\title{
A refined mass distribution of the cluster MACS J0416.1-2403 from a new large set of spectroscopic multiply lensed sources ${ }^{\star}$
}

\author{
G. B. Caminha ${ }^{1}$, C. Grillo ${ }^{2,3}$, P. Rosati ${ }^{1}$, I. Balestra ${ }^{4,5}$, A. Mercurio ${ }^{6}$, E. Vanzella ${ }^{7}$, A. Biviano ${ }^{5}$, K. I. Caputi ${ }^{8}$, \\ C. Delgado-Correal ${ }^{1}$, W. Karman ${ }^{8}$, M. Lombardi ${ }^{2}$, M. Meneghetti ${ }^{7,9}$, B. Sartoris ${ }^{10}$, and P. Tozzi ${ }^{11}$ \\ 1 Dipartimento di Fisica e Scienze della Terra, Università degli Studi di Ferrara, via Saragat 1, 44122 Ferrara, Italy \\ e-mail: mailto:gbcaminha@fe.infn.it \\ 2 Dipartimento di Fisica, Università degli Studi di Milano, via Celoria 16, 20133 Milano, Italy \\ 3 Dark Cosmology Centre, Niels Bohr Institute, University of Copenhagen, Juliane Maries Vej 30, 2100 Copenhagen, Denmark \\ ${ }^{4}$ University Observatory Munich, Scheinerstrasse 1, 81679 Munich, Germany \\ 5 INAF-Osservatorio Astronomico di Trieste, via G. B. Tiepolo 11, 34143 Trieste, Italy \\ 6 INAF-Osservatorio Astronomico di Capodimonte, via Moiariello 16, 80131 Napoli, Italy \\ 7 INAF-Osservatorio Astronomico di Bologna, via Ranzani 1, 40127 Bologna, Italy \\ Kapteyn Astronomical Institute, University of Groningen, Postbus 800, 9700 AV Groningen, The Netherlands \\ 9 INFN-Sezione di Bologna, viale Berti Pichat 6/2, 40127 Bologna, Italy \\ 10 Dipartimento di Fisica, Università degli Studi di Trieste, via G. B. Tiepolo 11, 34143 Trieste, Italy \\ 11 INAF-Osservatorio Astrofisico di Arcetri, Largo E. Fermi, 50125 Firenze, Italy
}

Received 12 July 2016 / Accepted 13 December 2016

\begin{abstract}
We report the spectroscopic confirmation of 22 new multiply lensed sources behind the Hubble Frontier Field (HFF) galaxy cluster MACS J0416.1-2403 (MACS 0416), using archival data from the Multi Unit Spectroscopic Explorer (MUSE) on the VLT. Combining with previous spectroscopic measurements of 15 other multiply imaged sources, we have obtained a sample of 102 secure multiple images with measured redshifts, the largest to date in a single strong lensing system. The newly confirmed sources are largely lowluminosity Lyman- $\alpha$ emitters with redshift in the range [3.08-6.15]. With such a large number of secure constraints, and a significantly improved sample of galaxy members in the cluster core, we have improved our previous strong lensing model and obtained a robust determination of the projected total mass distribution of MACS 0416. We find evidence of three cored dark-matter halos, adding to the known complexity of this merging system. The total mass density profile, as well as the sub-halo population, are found to be in good agreement with previous works. We update and make public the redshift catalog of MACS 0416 from our previous spectroscopic campaign with the new MUSE redshifts. We also release lensing maps (convergence, shear, magnification) in the standard HFF format.
\end{abstract}

Key words. galaxies: clusters: individual: MACS J0416.1-2403 - gravitational lensing: strong - cosmology: observations

\section{Introduction}

The use of gravitational lensing by galaxy clusters has intensified in recent years and has led to significant progress in our understanding of the mass distribution in clusters, as well as to the discovery of some of the most distant galaxies (e.g., Coe et al. 2013; Bouwens et al. 2014) thanks to the magnification of selected cluster lenses. Key to this progress has been the combination of homogeneous multi-band surveys of a sizeable number of massive clusters with the Hubble Space Telescope (HST), primarily with the Cluster Lensing And Supernova survey with Hubble (CLASH, Postman et al. 2012), with wide-field imaging (e.g., Umetsu et al. 2014, 2016) and spectroscopic follow-up work from the ground and space. Studies with HST have inevitably focused on the cluster cores, where a variety of strong lensing models have been developed to cope with the increasing data quality and to deliver the precision needed to determine the physical properties of background lensed galaxies (such as stellar masses, sizes and star formation

\footnotetext{
* Source catalog (full Table A.2) is only available at the CDS via anonymous ftp to cdsarc.u-strasbg. fr (130.79.128.5) or via http://cdsarc.u-strasbg.fr/viz-bin/qcat?J/A+A/600/A90
}

rates), which critically depend on the magnification measurement across the cluster cores. Following the CLASH project, which has provided a panchromatic, relatively shallow imaging of 25 massive clusters, the Hubble Frontier Fields (HFF) program (Lotz et al. 2016) has recently targeted six clusters (three in common with CLASH) to much greater depth ( $2 \mathrm{mag})$ in seven optical and near-IR bands with the ACS and WFC3 cameras. This has provided a very rich legacy data set to investigate the best methodologies to infer mass distributions of the inner $(R \lesssim$ $300 \mathrm{kpc}$ ) regions of galaxy clusters, and is stimulating a transition to precision strong lensing modeling with parametric (e.g., Richard et al. 2014; Jauzac et al. 2015b; Limousin et al. 2016; Kawamata et al. 2016) and non-parametric lens models (e.g., Lam et al. 2014; Diego et al. 2016; Wang et al. 2015; Hoag et al. 2016).

Spectroscopic follow-up information on a large number of multiply lensed sources is critical to achieve high-precision cluster mass reconstruction through strong lensing modeling. Early works heavily relied on photometric redshifts or color information to identify multiple images. While this method has been shown to be adequate for determining robust mass density profiles (e.g., Zitrin et al. 2015), it is prone to systematics due to 
possible misidentifications of multiple images and degeneracies between angular diameter distances and the cluster mass distribution. This typically leads to root-mean-square offsets $\left(\Delta_{\mathrm{rms}}\right)$ between the observed and lens model-predicted positions of $\Delta_{\text {rms }} \gtrsim 1^{\prime \prime}$ (see Zitrin et al. 2015, for the CLASH sample). Using extensive redshift measurements for both cluster member galaxies and background lensed galaxies, high-fidelity mass maps can be obtained with $\Delta_{\mathrm{rms}} \approx 0 \prime 3$, as shown for example, in the study of the HFF clusters MACS J0416.3-2403 (hereafter MACS 0416; Grillo et al. 2015, hereafter Gr15) and MACS J1149.5+2223 with the sucessful prediction of the lensed supernova Refsdal (Treu et al. 2016; Grillo et al. 2016).

Exploiting these new high-quality spectroscopic data sets in clusters that are relatively free from other intervening line-ofsight structures, strong lensing modeling even becomes sensitive to the adopted cosmology (Caminha et al. 2016, hereafter Ca16). In addition, new large spectroscopic samples of cluster member galaxies over a sufficiently wide area allow the cluster total mass to be derived based on galaxy dynamics (e.g., Biviano et al. 2013). This provides an independent, complementary probe of the cluster mass out to large radii, which, when combined with high-quality weak-lensing determinations, can in principle be used to infer dark-matter properties (Sartoris et al. 2014) or to test modified theories of gravity (Pizzuti et al. 2016).

The combination of photometric and spectroscopic data now available for MACS 0416, from extensive HST and VLT observations, makes it one of the best data sets with which to investigate the dark-matter distribution in the central region of a massive merging cluster through strong lensing techniques and to unveil high-redshift magnified galaxies owing to its large magnification area. The high-precision strong lensing model of MACS 0416 presented by Gr15 was based on CLASH imaging data and spectroscopic information obtained as part of the CLASH-VLT survey, presented in Balestra et al. (2016).

MACS 0416 is a massive and X-ray luminous $\left(M_{200} \approx\right.$ $0.9 \times 10^{15} M_{\odot}$ and $L_{\mathrm{X}} \approx 10^{45} \mathrm{erg} \mathrm{s}^{-1}$, Balestra et al. 2016) galaxy cluster at $z=0.396$, originally selected as one of the five clusters with high magnification in the CLASH sample. This system was readily identified as a merger, given its unrelaxed X-ray morphology and the observed projected separation $(\sim 200 \mathrm{kpc})$ of the two brightest cluster galaxies (BCGs; see Mann \& Ebeling 2012). Zitrin et al. (2013) performed the first strong lensing analysis using the available CLASH HST photometry, which revealed a quite elongated projected mass distribution in the cluster core $(\sim 250 \mathrm{kpc})$. In subsequent works Jauzac et al. (2014, 2015a) combined weak and strong lensing analyses, detecting two main central mass concentrations. When comparing their mass reconstruction with shallow Chandra observations, they were not able to unambiguously discern between a pre-collisional or post-collisional merger.

The CLASH-VLT spectroscopic sample of about 800 cluster member galaxies out to $\sim 4 \mathrm{Mpc}$ has recently allowed a detailed dynamical and phase-space distribution analyses, which revealed a very complex structure in the cluster core (Balestra et al. 2016). The most likely scenario, supported also by deep X-ray Chandra observations and VLA radio data, suggests a merger composed of two main subclusters observed in a pre-collisional phase.

In this work, we present a further improved strong lensing model of MACS 0416, which exploits a new unprecendeted sample of more than 100 spectroscopically confirmed multiple images (corresponding to 37 multiply imaged sources) and $\sim 200$ cluster member galaxies in the cluster core. In Sect. 2, we describe the MUSE spectroscopic data set, the data reduction procedure and the method used for redshift measurements. In Sect. 3, we describe the strong lensing model and discuss the results of our strong lensing analysis. In Sect. 4, we summarize our conclusions.

Throughout this article, we adopt a flat $\Lambda \mathrm{CDM}$ cosmology with $\Omega_{\mathrm{m}}=0.3$ and $H_{0}=70 \mathrm{~km} \mathrm{~s}^{-1} / \mathrm{Mpc}$. In this cosmology, $1^{\prime \prime}$ corresponds to a physical scale of $5.34 \mathrm{kpc}$ at the cluster redshift $\left(z_{\text {lens }}=0.396\right)$. All magnitudes are given in the AB system.

\section{Data}

In this work, we take advantage of the enhanced imaging data from the HFF campaign (Lotz et al. 2016) and significantly augment the CLASH-VLT wide-field spectroscopic campaign of MACS 0416 (Balestra et al. 2016) with a large number of new spectroscopic redshifts obtained with the MUSE integral-field spectrograph at the VLT, over the central area of $2 \operatorname{arcmin}^{2}$. The latter has led us to identify more than three times the number of secure multiple images with spectroscopic redshift used in Gr15 and two times when comparing with Hoag et al. (2016), as well as to define a highly complete and pure sample of cluster members. Details on MUSE data reduction and analysis are given in Sect. 2.1.

\subsection{MUSE observations and data reduction}

We used archival MUSE (Bacon et al. 2012) data from two different programs which covered the North-East (NE) and SouthWest (SW) regions of MACS 0416. The footprints of these two MUSE pointings are shown in Fig. 2 (see the magenta squares), overlaid onto the HST color image of the cluster. The NE region was observed within a GTO program (ID 094.A-0115B, PI: J. Richard) in November 2014, for a total of two hours split into four exposures. Significantly deeper observations in the SW region of the cluster were carried out by the program ID 094.A0525(A) (PI: F.E. Bauer). The latter includes 58 exposures of approximately 11 minutes each, executed over the period October 2014-February 2015. In both programs, each exposure was offset by fractions of arcseconds and rotated by 90 degrees to improve sky subtraction. The seeing conditions of the NE pointing were very good, $\approx 0 \prime .5$, based on the DIMM monitor at Paranal (the lack of bright stars in both pointings did not allow us to directly measure the seeing on MUSE data). Most of the exposures of the SW pointing, 30 out of 58, were taken in seeing conditions $<1^{\prime \prime}$, whereas the others have $F W H M \simeq 1^{\prime \prime}-1^{\prime \prime} .3$. Moreover, a visual inspection of all SW exposures from the stacked data-cubes did not show evidence of significant variations in observational conditions. Only one exposure was discarded due to the presence of a satellite track, leading to a total of $11 \mathrm{~h}$ of exposure time in the SW pointing.

We used the MUSE reduction pipeline version 1.2.1 to process the raw calibration and science exposures of each single night, and to combine the data into the two final data-cubes. During this process we applied all the standard calibration procedures (bias and flat field corrections, wavelength and flux calibration, etc.) provided by the pipeline. We then combined the observing blocks of the NE and SW observations (taking into account the offset of each observation) into two final data-cubes. Different configurations of the MUSE pipeline recipes were experimented to improve the quality of the final data-cubes, particularly the sky subtraction, with no significant differences however. The final WCS adjustment was made matching compact sources detected with SExtractor (Bertin \& Arnouts 1996) in the 
broad-band images of the two final data-cubes with the corresponding objects in the HFF catalog for the filter $F 606 \mathrm{~W}$. As a final post-processing step to minimize the sky residuals, we applied the Zurich Atmosphere Purge (ZAP, Soto et al. 2016) tool using SExtractor segmentation maps to define sky regions.

The two final data-cubes have a spatial pixel scale of 0 '.2, a spectral coverage from $4750 \AA$ to $9350 \AA$, with a dispersion of $1.25 \AA /$ pixel and a fairly constant spectral resolution of $\approx 2.4 \AA$ over the entire spectral range. We noticed that after using different configurations of the MUSE pipeline and applying the ZAP tool, an overall improvement was achieved in the sky subtraction even though artefacts in the background at specific wavelengths still remain particularly in the SW pointing, due to residual instrumental signatures and sky subtraction. Nonetheless, the quality of the reduced data-cubes allows the spectroscopic identifications of approximately one hundred sources in each pointing, reaching very faint levels as described below.

We notice that despite the significant longer exposure, the signal-to-noise of spectra in the SW pointing does not scale according to expectations, resulting only in a moderately larger depth when compared to the NE pointing. We attribute this difference to the significantly better seeing of the NE pointing $\left(0^{\prime \prime} .5\right.$ versus $1^{\prime \prime}$ ) and the large number of short exposures used in the observations of the SW pointing, which due to residual systematics in the background subtraction, did not yield the expected depth in the coadded datacube.

\subsection{Spectra extraction and redshift measurements}

We describe here the strategy and methodology to extract spectra and measure redshifts for all detectable objects in the MUSE fields, specifically cluster members and multiple images of background lensed sources, which are critical inputs of our strong lensing model.

To maximize the completeness of spectroscopic identifications in the two MUSE data-cubes we proceeded in two steps. Firstly, we used the ASTRODEEP Frontier Fields catalog by Castellano et al. (2016), and blindly extracted spectra at each object position from the MUSE data-cubes, whose world coordinate system is aligned with the HFF within $\sim 00^{\prime \prime} 1$. Spectra were extracted within 0":8-radius circular apertures, which provide a good compromise in the effort to maximize signal-tonoise and minimize source confusion. The ASTRODEEP HFF catalog reaches a $90 \%$ completeness limit at $\operatorname{mag}_{F 160 W} \approx 27.25$ for disk-like galaxies. Objects which are flagged as possible spurious detections in the ASTRODEEP catalog were not considered. In total, we extracted 716 and 699 spectra in the SW and NE MUSE pointings, respectively.

Two team members used the software EZ (Garilli et al. 2010) and SpecPro (Masters \& Capak 2011) to measure independently redshifts, assigning quality flags following the scheme described in Caminha et al. (2016) and Balestra et al. (2016), that is $3=$ secure, $2=$ likely, $1=$ not-reliable and $9=$ based on a single emission line. Since the MUSE spectral resolution allows us to distinguish the shape or doublet nature of narrow emission lines (Lyman- $\alpha$ and O II, for instance), the redshifts with quality flag equal to 9 are considered very reliable. With this procedure, we measured $\approx 300$ reliable redshifts with quality flag greater than 1. Approximately one-third of the objects show emission lines in their spectra.

As an additional step, we visually inspected the original and continuum-subtracted cubes. Continuum subtraction was obtained at each wavelength frame by considering two windows, ten spectral pixels $(=12.5 \AA$ ) wide, in the blue and red side of each frame, separated by ten spectral pixels. The continuum is estimated from the mean of the median counts in each of these two regions and then subtracted from each wavelength slice. The inspection of such a continuum subtracted data-cube allowed us to identify faint emission lines of sources close to bright galaxies. In this way, we identified 14 additional faint sources, mostly Lyman- $\alpha$ emitters, with very faint or non-detectable counterpart in the HFF images.

In summary, the analysis of the available MUSE observations of MACS 0416 led us to extend the previous redshift catalog from the CLASH-VLT survey, published in Balestra et al. (2016), which now contains 301 MUSE based redshifts, of which 208 are new. With this paper, we also electronically release the updated version of the redshift catalog, combining the VIMOS and MUSE observations, which now contains approximately 4600 objects with redshifts (quality flag greater than 1). A direct comparison between MUSE and VIMOS spectra can be done with Figs. A. 1 and 2 of Gr15.

The GLASS survey (Treu et al. 2015) has provided redshift measurements of 170 sources in an area of $\approx 4 \operatorname{arcmin}^{2}$ around the cluster core. We have found that 103 redshifts from the CLASH-VLT and MUSE data sets are in common with GLASS. Within the MUSE field of view, we were not able to measure redshifts for ten GLASS sources, of which six are close to the edge of the MUSE observations. The remaining four have redshifts below $z=2.9$, the lower limit of a Lyman- $\alpha$ emission to appear in the MUSE spectrum.

Regarding the identification of multiple images for the strong lensing model described below, in this work we confirm 11 of the 15 spectroscopic multiple-image families from previous studies (Richard et al. 2014; Johnson et al. 2014; Jauzac et al. 2014; Grillo et al. 2015; Hoag et al. 2016) and measure secure redshifts for additional 56 multiple images, belonging to 21 new families, thus more than doubling the number of multiple images with spectroscopic redshift known to date for MACS 0416. We also measure the redshift of additional five multiple images belonging to known spectroscopic families but with no previous spectroscopic confirmation. Finally, we update the redshift of family 1 to $z=3.238$, previously reported in the GLASS catalog at $z=2.19$ with quality flag "probable" in their definition. We therefore spectroscopically determine with MUSE a total of $21+1$ new multiply lensed systems. All except one of these 37 families have at least two images with measured redshift. All these multiple images are indicated in Fig. 1, while spectra and image cutouts are shown in Fig. A.1. Many of these lensed sources have faint magnitudes for ordinary ground-based spectroscopic work, ranging from $\operatorname{mag}_{F 814 W}=24$ down to $\approx 29$ (see Table A.1) and redshifts $z \gtrsim 3.08$ (reflecting the visibility of the Lyman- $\alpha$ in the MUSE window). These sources are primarily low-luminosity Lyman- $\alpha$ emitters, whose spectro-photometric properties can be used to constrain physical properties of lowmass galaxies which are considered to be the main candidates for reionization (Wise et al. 2014; Kimm \& Cen 2014). Moreover, the rest-frame equivalent widths of these lensed Lyman$\alpha$ emitters range between $\approx 10 \AA$ to $\approx 120 \AA$ and have extremely low luminosities $\left(L \approx 10^{40} L_{\odot}-10^{42} L_{\odot}\right)$, which are comparable to those measured in the sample of lensed LAEs in Karman et al. (2017).

When combined with previous measurements, from the CLASH-VLT and GLASS surveys, the multiple-image systems span a redshift range from $z \approx 0.94$ up to $z \approx 6.15$. The highest redshift source is a remarkable giant arc with three multiple images $(2 \mathrm{a}, 2 \mathrm{~b}$ and $2 \mathrm{c})$, as revealed by the MUSE data-cube at the 


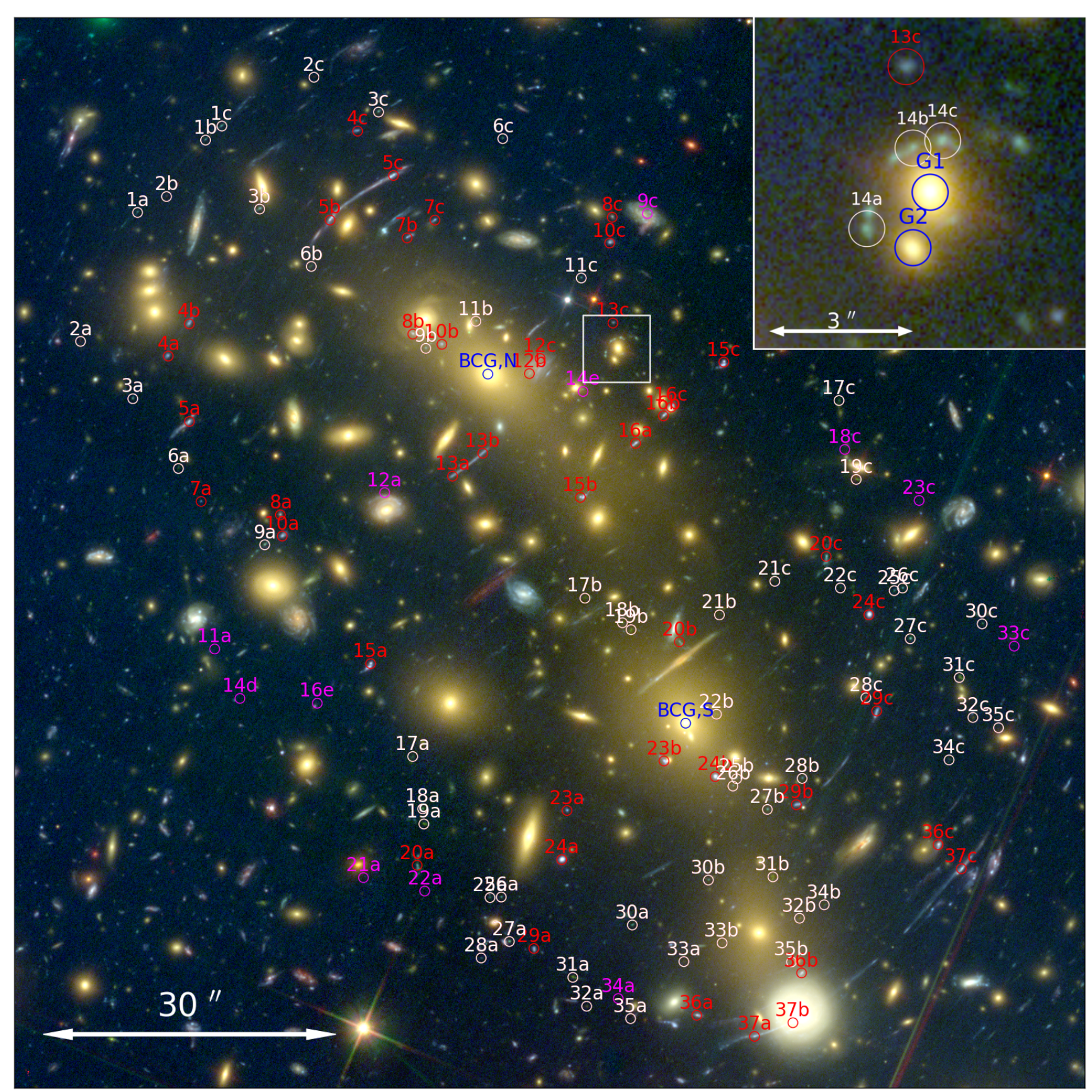

Fig. 1. Composite image of MACS 0416 from Hubble Frontier Fields data. Blue, green and red channels are the combination of filters $F 435 W$, $F 606 W+F 814 W$ and $F 105 W+F 125 W+F 140 W+F 160 W$, respectively. White circles mark the positions of the 59 multiple images belonging to 22 families with new spectroscopic confirmation in this work, while red circles show multiple images previously known in spectroscopic families. Magenta circles show the model-predicted positions of multiple images not included in our model, lacking secure identifications (see Table A.1). The inset is a blow-up of the region around family 14, around two galaxy cluster members, G1 and G2, with total mass density profile parameters free to vary in our model (see Sect. 3). The blue circles indicate the positions of the BCGs (BCG, N and BCG, S).

$8686 \AA$ A. A very interesting multiply imaged system is System 9, which consists of a complex, double-peaked, extended Lyman- $\alpha$ emission in which three faint galaxies, detected on the HST image, are embedded. This system has been studied in detail in Vanzella et al. (2017). We also note that two families (IDs 21 and 35) do not have significant counterparts in the deep HFF images (see Table A.1), showing the remarkable ability that MUSE has in identifying very faint emission line objects.

In Fig. 3, we compare the number of spectroscopically confirmed multiple-image families and physical magnification areas for all HFF clusters, as well as for Abell 1689, which has long been a reference cluster for high-quality lensing studies (in this case, we computed the magnification area using the public lens model in Limousin et al. 2007). The error bars show the variance of the results of the different strong lensing models that can be found on the HFF webpage ${ }^{1}$. For Abell 1689, only one strong lensing model is publicly available, so no error bars are provided. These two parameters enter a figure of merit for the quality of the reconstructed mass distribution of a cluster, since the fidelity of the mass map strictly depends on the number of multiple images

1 https://archive.stsci.edu/prepds/frontier/ lensmodels/ 


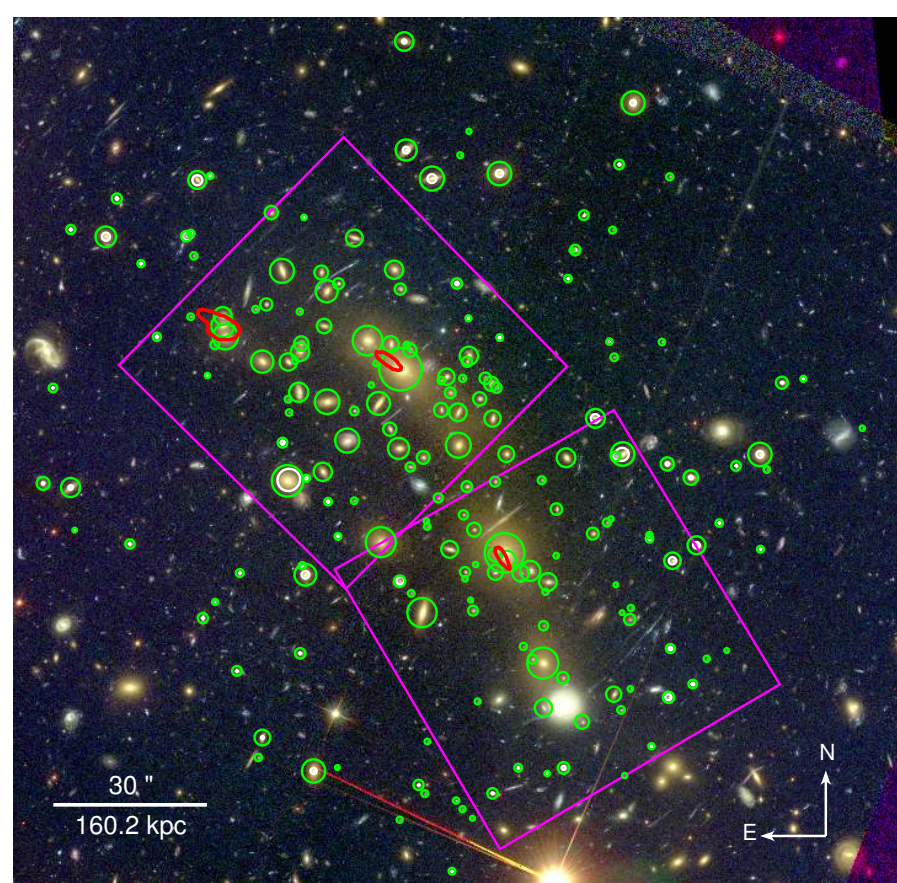

Fig. 2. Image of MACS 0416 from the Hubble Frontier Fields data (using the ACS filters $F 435 W, F 606 W$ and $F 814 W$ for the blue, green and red channels respectively) with the two overlaid MUSE pointings (magenta boxes), each $\approx 1^{\prime}$ across. The green circles indicate the 193 selected galaxy cluster members $(75 \%$ of which spectroscopically confirmed), with the radii proportional to the values of $\sigma_{v}$ obtained from the best-fitting lensing model and using Eq. (1). The three red contours show the $95 \%$ confidence level of the dark-matter halo centers included in the lensing model.

Table 1. Comparison of strong lensing constraints, that is the number of multiple image families with spectroscopic confirmation $\left(N_{\text {spec }}\right)$, for the best-studied strong lensing clusters to date.

\begin{tabular}{lcc}
\hline \hline Cluster & $N_{\text {spec }}$ & Reference \\
\hline MACS 0416 & 37 & this work \\
MACS 0416 & 15 & Hoag et al. (2016) \\
MACS 0416 & 8 & Grillo et al. (2015) \\
\hline Abell 1689 & 24 & Diego et al. (2015a) \\
Abell S1063 & 17 & Karman et al. (2017) \\
MACS J1149.5+2223 & 8 & Treu et al. (2016) \\
Abell 2744 & 10 & Jauzac et al. (2015b) \\
MACS J0717.5+3745 & 9 & Limousin et al. (2016) \\
Abell 370 & 3 & Richard et al. (2010) \\
\hline
\end{tabular}

with spectroscopic redshift, while the spatial resolution of this map depends on the number of strong lensing constraints for a given extent of the magnification area.

In Table 1, we quote strong lensing constraints and references for the HFF clusters and Abell 1689. We show the progress in the identification of multiple-image families for MACS 0416, from Gr15 (using VLT/VIMOS), to Hoag et al. (2016, using HST/GLASS), to this work (with VLT/MUSE). The 21 additional spectroscopic multiply lensed sources presented here make MACS 0416 arguably the best-studied strong lensing cluster to date.

The MUSE data also allow us to extend and check the purity of the catalog of 175 cluster members presented in Gr15. In that work, 12 CLASH photometric bands were used to define

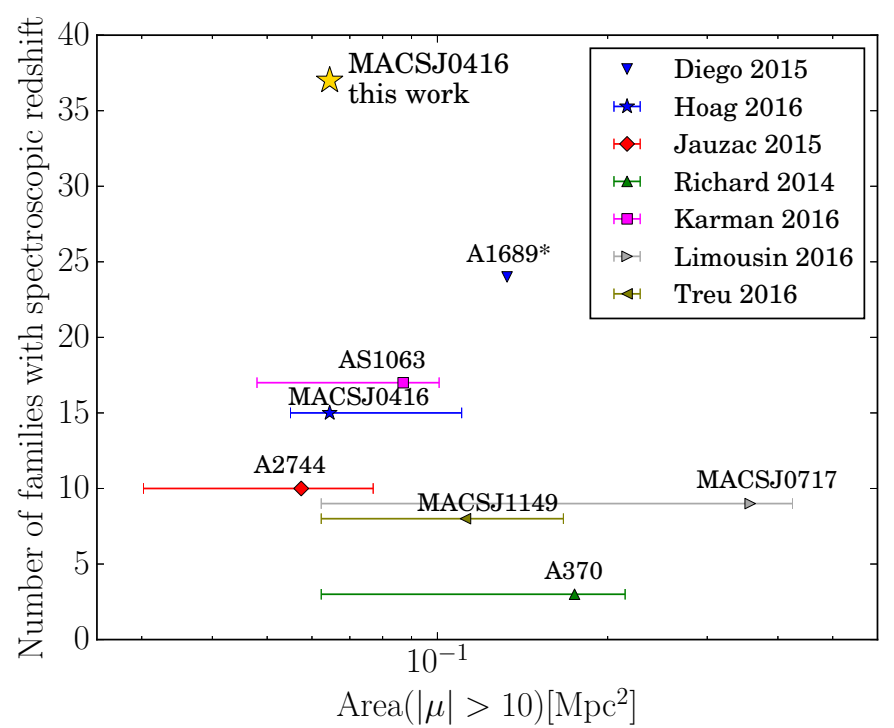

Fig. 3. Number of spectroscopically confirmed families of multiple images identified to date, as a function of the physical area on the lens plane with absolute magnification greater than 10 , for a source at $z=4$. In addition to the HFF clusters, we also include Abell 1689. The error bars of the magnification area reflect the variance (ninetieth percentile) of the most recent HFF strong lensing models.

the distribution in color space of spectroscopic members from the CLASH-VLT campaign, thereby assigning a probability to all other galaxies to be a member based on their $N$-dimensional color. We have already emphasized that the completeness and purity of the sample of sub-halos associated to cluster members play an important role in the quality of the strong lensing model.

Interestingly, we find that only four galaxies were misidentified as galaxy members in Gr15, and add 22 new spectroscopic members brighter than $\operatorname{mag}_{F 160 W}=24$. The latter is the limiting magnitude adopted in Gr15 to define the photometric sample within the HST/WFC3 FoV $\left(\approx 5.5 \operatorname{arcmin}^{2}\right)$. Following Gr15, we used the redshift range [0.382-0.410] to define membership. This interval, corresponding to approximately three times the cluster velocity dispersion in the rest frame, is somewhat wider than the velocity range of members selected with kinematic methods in Balestra et al. (2016). In Fig. 4, we show the magnitude distribution of the cluster members, highlighting the improvement enabled by the MUSE data, which provides also nine members fainter than $\operatorname{mag}_{F 160 W}=24$. This is not surprising, considering that MUSE observations outperform VIMOS spectroscopy (with 1-2 h exposure) when measuring redshifts of faint early-type galaxies, and that the Gr15 cluster member catalog was constructed maximizing purity over completeness. Thus, the new sample of cluster members inlcudes 193 galaxies, of which 144 (75\%) have measured spectroscopic redshifts. Finally, we note that all cluster members with measured GLASS redshifts are confirmed by MUSE.

\section{Strong lensing modeling}

We use the positions of the new large set of multiple images described above to study the mass distribution of MACS 0416 with the strong lensing technique described in Ca16 (see Sect. 3 of that paper for details and equations). We briefly summarize here the main characteristics of our strong lensing model. We use the public software lenstool (Kneib et al. 1996; Jullo et al. 2007) to reconstruct the projected total mass distribution in a parametric 


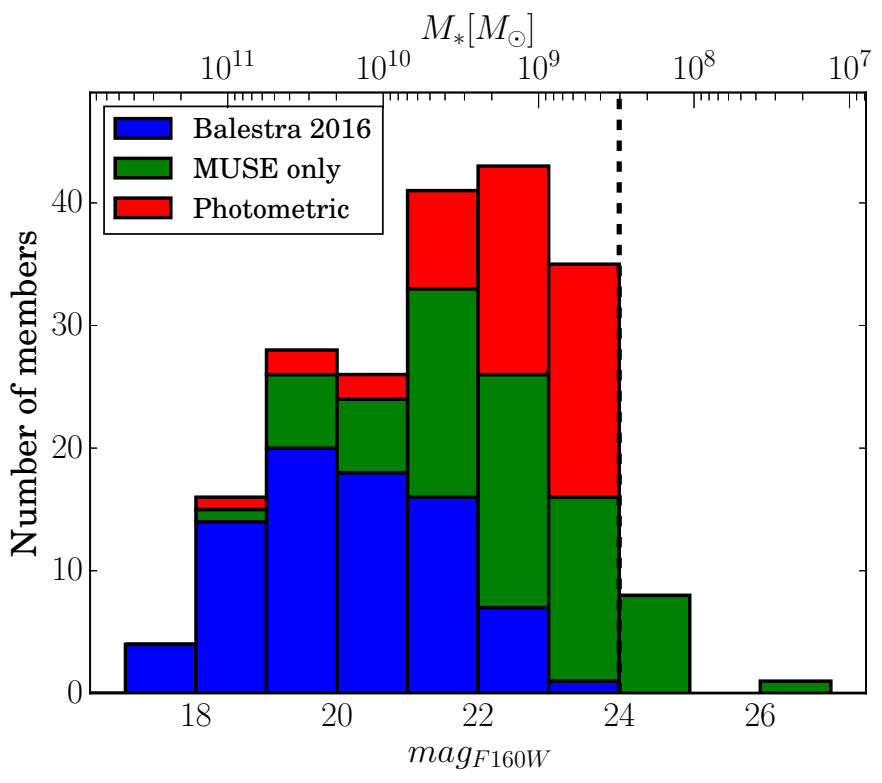

Fig. 4. Stacked distribution of $F 160 \mathrm{~W}$ magnitudes of galaxy members in the core of MACS 0416. Spectroscopically confirmed members from CLASH-VLT (Balestra et al. 2016) are shown in blue (80), the newly identified members by MUSE in green (73) and the remaining photometrically selected galaxies with $\operatorname{mag}_{F 160 W}<24$ from Gr15 in red (193 in total). The stellar mass on the top axis is computed from $\log \left(M_{*} / M_{\odot}\right)=18.541-0.416 \times \operatorname{mag}_{F 160 W}$ from Gr15.

form, by minimizing the distances between the model-predicted and observed positions of the multiple images.

As emphasized in Gr15 and Ca16, we only rely on secure multiple images with spectroscopic redshift, since additional photometric multiply lensed systems lead to systematic uncertainties in the model, due to possible misidentification of multiple images, and introduce degeneracies in the mass distribution, due to uncertain angular diameter distances associated with photometric or unknown redshfits. We select the new multiple images firstly from our spectroscopic catalog, identifying the sources with very similar redshifts. Only a few sources are found to be at the same redshift but are not multiple images. These sources likely belong to a proto-cluster or group of galaxies behind MACS 0416. Notice that we did not use our previous strong lensing model of $\mathrm{Gr} 15$ to select the new multiple image families. Our selection strongly relies on the spectroscopic identifications.

In order not to bias our model, we do not include any family without spectroscopic confirmation and do not exclude spectroscopically confirmed multiple images that cannot be reproduced well by our model. Moreover, we do not include multiple images when the identification is not secure, either because no detectable images or spectroscopic emission is found, or the image association is not unique (see magenta circles in Fig. 1). Specifically, we find more than one candidate image at the location where we expected to find the multiple images 9c, 11a, 14d, 16e, $18 \mathrm{c}$ and $23 \mathrm{c}$, making their identification uncertain. In the case of multiple image $12 \mathrm{a}$, its strong distortion due to a galaxy member does not allow us to identify the luminosity peak corresponding to the images $12 \mathrm{~b}$ and $12 \mathrm{c}$. Moreover, we do not find clear counterparts of 21a, 22a, 33c and 34a in the HFF imaging. On the other hand, although we do not have spectroscopic confirmation of the multiple images $17 \mathrm{~b}, 17 \mathrm{c}$ and $28 \mathrm{a}$, their color, morphology and parity are in very good agreement with other images with measured redshifts, resulting in secure multiple-image systems.
Interestingly, the families 21 and 35 show very clear Lyman- $\alpha$ emission but no evident counterparts in the HFF imaging. For these two families, we consider multiple-image positions at the peaks of the emission in the MUSE data. In summary, we build a secure set of 102 multiple images (belonging to 37 families) with spectroscopic redshifts, to reconstruct the total mass distribution of MACS 0416. The coordinates, redshifts, magnitudes, previous literature information of the multiple images in our final set are presented in Table A.1.

We adopt an uncertainty on the observed multiple image positions $\left(\sigma^{\text {obs }}\right)$ of $0^{\prime \prime} .5$, which takes into account possible perturbations form line-of-sight structures, as discussed in Gr15 and Ca16 and close to the theoretical expectations (Jullo et al. 2010; Host 2012). We show below that this value yields a reduced $\chi^{2}$ value very close to one. To compute the posterior probability distributions of the model parameters, hence their statistical errors and correlations, lenstool uses a Bayesian Markov chain Monte Carlo (MCMC) technique.

\subsection{Mass model components}

The overall total mass distribution of the cluster is modeled with a smooth component, made of one or more halos which represent the dominant dark matter, the hot gas and the intra-cluster light, and a clumpy sub-halo population traced by the member galaxies.

Each smooth component is parametrized with a pseudoisothermal elliptical mass distribution (PIEMD; Kassiola \& Kovner 1993). This model is characterized by the values of an effective velocity dispersion $\left(\sigma_{v}\right)$, core radius $\left(r_{\text {core }}\right)$, ellipticity $(\varepsilon)$ and position angle $(\theta)$. The ellipticity is defined as $\varepsilon \equiv\left(a^{2}-b^{2}\right) /\left(a^{2}+b^{2}\right)$, where $a$ and $b$ are the semi-major and minor axis, respectively. Since the distribution of dark matter is not necessarily associated to an observable counterpart, the center of this component ( $x$ and $y$ ) is also a free parameter in the model.

As for the clumpy component, we exploit the new highly complete sample of cluster members described above, attaching a halo to each of the 193 member galaxies. Each halo is modeled with a circular pseudo-isothermal mass distribution (dPIE; Elíasdóttir et al. 2007; Suyu \& Halkola 2010). The position of each dPIE is fixed at the luminosity center of member galaxies, while the values of effective velocity dispersion $\sigma_{v, i}^{\text {gals }}$ and truncation radius $r_{\text {cut }, i}^{\text {gal }}$ are free parameters. Following Gr15 anf Ca16, we scale these parameters with the observed luminosity in the filter $F 160 W$ of each galaxy, using:

$$
\sigma_{v, i}^{\mathrm{gals}}=\sigma_{v}^{\mathrm{gals}}\left(\frac{L_{i}}{L_{0}}\right)^{0.35} \text { and } r_{\mathrm{cut}, i}^{\mathrm{gals}}=r_{\text {cut }}^{\mathrm{gals}}\left(\frac{L_{i}}{L_{0}}\right)^{0.5},
$$

where $L_{0}$ is a reference luminosity which we choose to coincide with that of the northern BCG (BCG, $\mathrm{N}$ in Fig. 1, $\operatorname{mag}_{F 160 \mathrm{~W}}=$ 17.02). We are thus left with only two parameters ( $\sigma_{v}^{\text {gals }}$ and $\left.r_{\text {cut }}^{\text {gals }}\right)$ describing the overall sub-halo population. These specific relations yield a shallow dependence of the galaxy total mass-tolight ratio with their luminosity, that is $M_{\text {total }} / L \propto L^{0.2}$. Such a "tilted" scaling relation is known from studies on the Fundamental Plane (Faber et al. 1987; Bender et al. 1992) of earlytype galaxies and has been shown to better reproduce the observed positions of multiple images in previous high-precision lensing models, such as those for MACS 0416 itself (Gr15) and MACS J1149.5+2223 (Grillo et al. 2016), when predicting the reappearance of SN Refsdal (Kelly et al. 2016). 
The inset in Fig. 1 shows a galaxy-scale lensing system (family 14) embedded in the cluster potential. Since the cluster galaxies G1 and G2 are the main contributors to the creation of the multiple images of this system, the total mass density parameters of these two galaxies are left free to vary in the modeling. For the more luminous galaxy G1, we consider an elliptical profile and optimise also its values of ellipticity and position angle. We therefore have six extra free parameters describing G1 and $\mathrm{G} 2:\left(\sigma_{v}^{\mathrm{G} 1}, r_{\text {cut }}^{\mathrm{G} 1}, \varepsilon_{\mathrm{G} 1}, \theta_{\mathrm{G} 1}\right)$ and $\left(\sigma_{v}^{\mathrm{G} 2}, r_{\text {cut }}^{\mathrm{G} 2}\right)$.

Similarly to previous works (Gr15; Johnson et al. 2014; Richard et al. 2014; Kawamata et al. 2016), we take into account the lensing contribution of a foreground galaxy at $z=0.112$ located in the South-West region of MACS 0416, very close to family 37 (see Fig. 1). To do that, we include an extra dPIE mass component at the galaxy position ( $\mathrm{RA}=04: 16: 06.82$ and Dec $=-24: 05: 08.4)$ and cluster redshift, making its "effective" parameters $\sigma_{v}^{\text {fore }}$ and $r_{\text {cut }}^{\text {fore }}$ also free to vary. This is a first simple approximation to a correct multi-plane lensing model, which is not included yet in the lenstool software.

\subsection{Results}

Following Gr15, we first use two halos for the smooth total mass component of MACS 0416, a complex merging cluster, as clearly indicated by the distribution of cluster galaxies in two main clumps around the BCG North and South. This model has a total of 22 free parameters describing the cluster total mass distribution and can reproduce the observed positions of the multiple images with values of root-mean-square offset $\left(\Delta_{\mathrm{rms}}\right)$ of 0 "':82 and minimum $\chi^{2}$ of 275 . In this case, the number of degrees of freedom (i.e., the number of observables minus the number of free parameters) is 108 , resulting in a reduced $\chi^{2}$ of 2.55 , significantly higher than what was found in previous strong lensing studies on this cluster.

By inspecting the spatial distribution of the offsets between the observed and model-predicted positions of the multiple images, we notice larger offsets in the region NE of the northern BCG. We then add a third extended spherical pseudo-isothermal halo with four free parameters, namely $\sigma_{v 3}, r_{\text {core, } 3}$ and its center $\left(x_{3}, y_{3}\right)$ free to vary across the entire FoV. We find that the addition of this third halo reduces significantly the $\Delta_{\mathrm{rms}}$ and $\chi^{2}$ values to $00^{\prime \prime} 59$ and 143 , respectively, and that the best-fitting center of this extra component is very close to a relatively minor clump in the galaxy distribution (see Fig. 2). Interestingly, such a clump was not selected as an overdensity in the phase-space analysis of cluster galaxies over the entire cluster by Balestra et al. (2016), however its center is in good agreement with a peak in the convergence map obtained by Hoag et al. (2016, the offset is only $\approx 5^{\prime \prime} 5$, see their Fig. 5), who performed a free form reconstruction of the total mass distribution of MACS 0416, combining both weak and strong lensing.

In summary, the cluster total mass distribution adopted here has 26 free parameters: 1) 16 describing the three smooth darkmatter components; 2) six describing the galaxy-scale lensing system; 3) two for the prominent foreground galaxy; and 4) two describing the mass-luminosity scaling relation of galaxy members. Overall, the number of constraints from the positions of the 102 multiple images associated to 37 sources is 130 $(102 \times 2-37 \times 2)$, and therefore the number of degrees of freedom is $104(130-26)$. Notably, these constraints are well distributed across the entire central region of MACS 0416, spanning a wide redshift range (0.94-6.15), which is key to the fidelity of the reconstructed cluster total mass distribution and break the masssheet degeneracy (Falco et al. 1985; Seitz \& Schneider 1997).
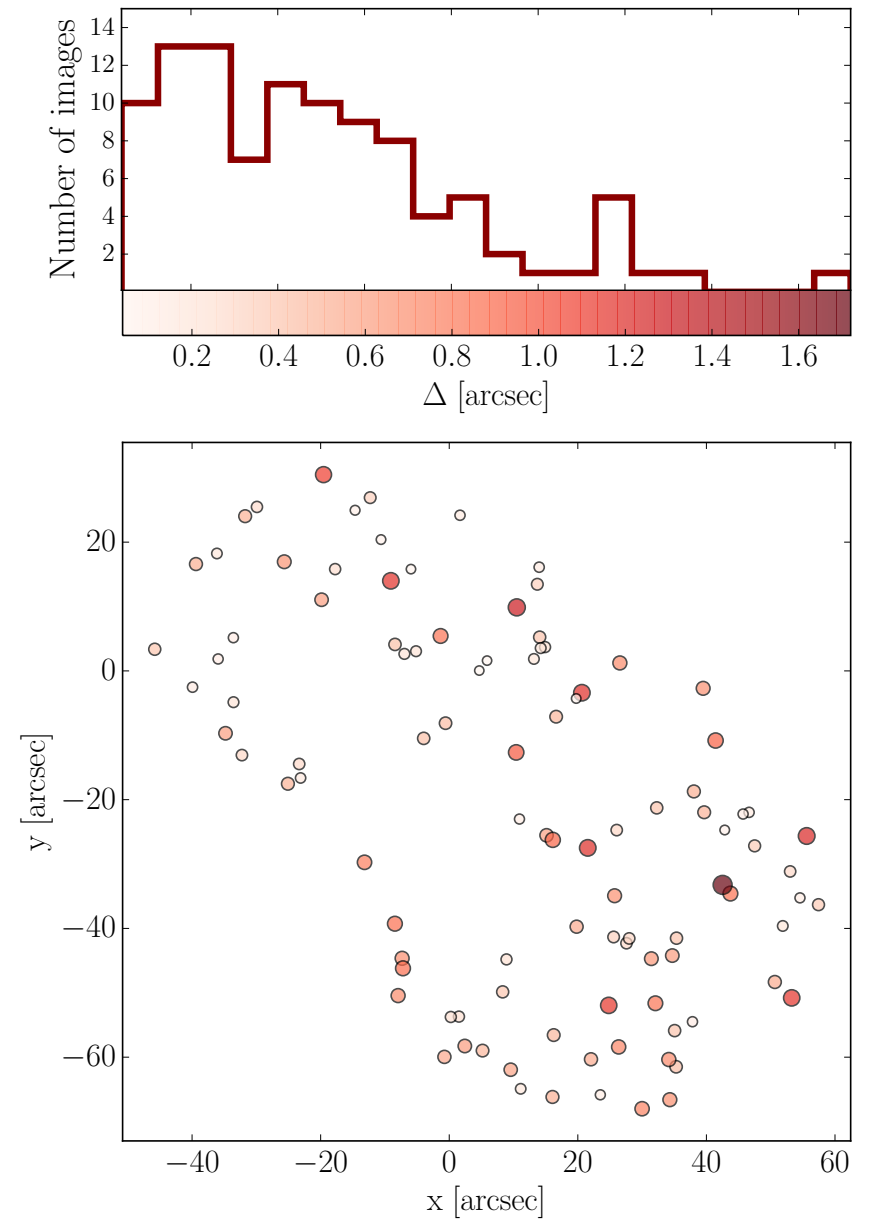

Fig. 5. Top panel: distribution of the absolute value of the offsets $\Delta$ between the observed and predicted (from our best-fitting model) positions of the multiple images. Bottom map: spatial distribution of these offsets. The circles indicate the positions of the observed multiple images (relatively to the northern BCG), with colors and sizes scaling with their positional offsets.

In Table 2, we show the median values of the parameters of our strong lensing models with their intervals at the $68 \%, 95 \%$ and $99.7 \%$ confidence levels (CL). The coordinates are relative to the position of the northern BCG and the angles are counted counterclockwise from the horizontal axis. The final reduced $\chi^{2}$ value is close to one $(=1.37)$, while the rms offset is $\Delta_{\mathrm{rms}}=0^{\prime \prime} .59$ $\left(\Delta_{\text {median }}=0 \prime 5\right.$, see Fig. 5$)$.

The best-fitting centers of the three diffuse halos are shown as red contours (corresponding to a 95\% significance level) in Fig. 2. In Gr15, we discussed the apparent offest in the projected distance between the centers of the two main halos and the corresponding BCGs. We cautioned that while an offset of 9':3 and 5".8, relative to BCG, N and BCG, S respectively, was statistically significant, systematics inherent in the parametric form of the lens model, as well as projection effects, made it difficult to claim such an offset, which has often been used to constrain the collisionless nature of DM (Williams \& Saha 2011; Kahlhoefer et al. 2015), between the DM and stellar component. Interestingly, our new model, which is based on $\approx 3$-times the number of multiple images of Gr15, albeit with an extra halo component, leads to a projected distance of only $3 \prime 0_{-2.6}^{+2.1}$ and $1 " 0_{-1.4}^{+0.5}(99.7 \% \mathrm{CL})$ of the two main halos from the BCG North and South, respectively. The statistical significance of the offset 
Table 2. Median values and confidence levels of the cluster total mass distribution parameters from the MCMC analysis of the strong lensing model.

\begin{tabular}{|c|c|c|c|c|}
\hline & Median & $68 \% \mathrm{CL}$ & $95 \% \mathrm{CL}$ & $99.7 \% \mathrm{CL}$ \\
\hline \multicolumn{5}{|c|}{ Halo around BCG-North (relative position $\left.=\left(0^{\prime \prime}, 0^{\prime \prime}\right)\right)$} \\
\hline$x_{1}\left({ }^{\prime \prime}\right)$ & -2.4 & ${ }_{-0.6}^{+0.7}$ & $\begin{array}{l}+1.4 \\
-1.2\end{array}$ & ${ }_{-1.8}^{+2.2}$ \\
\hline$y_{1}\left({ }^{\prime \prime}\right)$ & 1.8 & $\begin{array}{l}-0.0 \\
-0.5\end{array}$ & +0.8 & $\begin{array}{l}-1.0 \\
+1.2 \\
-1.8\end{array}$ \\
\hline$\varepsilon_{1}$ & 0.85 & $\begin{array}{l}-0.01 \\
-0.01\end{array}$ & +0.03 & +0.04 \\
\hline$\theta_{1}(\mathrm{deg})$ & 143.9 & $\begin{array}{l}-0.01 \\
+0.8\end{array}$ & $\begin{array}{l}-0.03 \\
+1.7\end{array}$ & $\begin{array}{l}-0.04 \\
+2.4\end{array}$ \\
\hline & & -0.8 & -1.7 & -2.5 \\
\hline$r_{\text {core }, 1}\left({ }^{\prime \prime}\right)$ & 6.3 & $\begin{array}{l}+0.7 \\
-0.7\end{array}$ & $\begin{array}{l}+1.3 \\
-1.3\end{array}$ & $\begin{array}{l}+1.9 \\
-1.9\end{array}$ \\
\hline$\sigma_{v 1}\left(\mathrm{~km} \mathrm{~s}^{-1}\right)$ & 707 & $\begin{array}{l}+26 \\
-28\end{array}$ & $\begin{array}{l}+50 \\
-56\end{array}$ & $\begin{array}{r}+79 \\
-83\end{array}$ \\
\hline
\end{tabular}

Halo around BCG-South (relative position $=\left(20\right.$ '!3, $\left.\left.-35^{\prime \prime} .8\right)\right)$

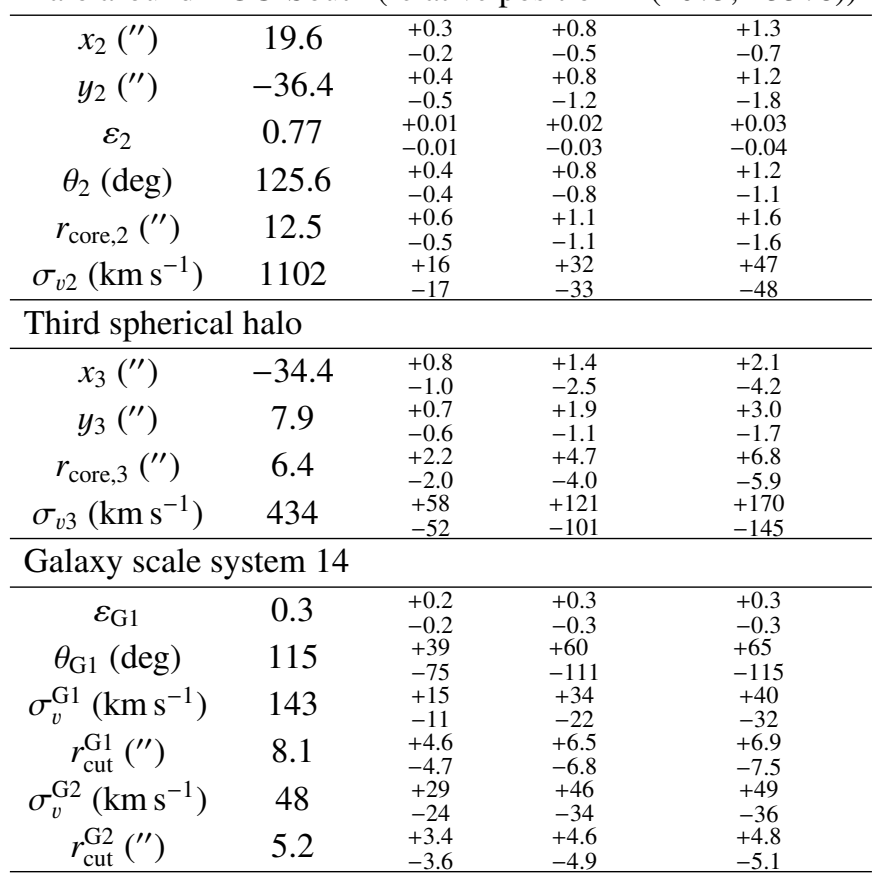

Sub-halo population

\begin{tabular}{ccccc}
\hline \multicolumn{2}{c}{ gals } & \\
$r_{\text {cut }}\left({ }^{\prime \prime}\right)$ & 10.5 & +2.7 & +6.2 & +10.3 \\
$\sigma_{v}^{\text {gals }}\left(\mathrm{km} \mathrm{s}^{-1}\right)$ & 251 & -2.4 & -4.6 & -6.2 \\
& & +14 & +31 & +48 \\
\hline
\end{tabular}

Notes. Coordinates are relative to the position of the BCG, N $(\mathrm{RA}=04: 16: 09.15$ and $\mathrm{Dec}=-24: 04: 03.0)$.

between each DM halo and its hosting BCG is therefore reduced when compared with Gr15. We defer a further analysis on this issue to a future paper, where the velocity dispersions of the BCGs are used to alleviate the degeneracy between the parameters (centers and scale) of the DM halos and those associated to the BCGs.

The center of the third dark matter halo $\left(x_{3}\right.$ and $\left.y_{3}\right)$ is on the top of a clump of three galaxy members in the north-east region of the cluster (see Fig. 2) and its mass is significantly smaller than that of the other two halos. However, the possibility of having a vanishing mass (i.e., $\sigma_{v 3}=0$ ) is excluded by the posterior distribution computed from the MCMC, statistically confirming the existence of this halo. Finally, within a circle with radius of $\approx 15^{\prime \prime}$ from this halo there is no evidence of a background or foreground structure in our redshift measurements and in the
HST imaging, thus excluding a possible existence in this region of a significant perturber not belonging to MACS 0416.

Regarding the galaxy-scale lensing system 14, it is worth noticing that although the value of the position angle of G1 is not well constrained (see Table 2), its median value is in very good agreement with that of the light distribution, $\theta_{\mathrm{G} 1}^{\text {light }}=119^{\circ}$, measured with SExtractor. On the other hand, the ellipticity is unconstrained due to degeneracies with the smooth dark-matter halos. Moreover, the values of effective velocity dispersion from the best-fitting scaling relation of the cluster members (using Eq. (1) and the MCMC chain used to compute the values in Table 2) are $108_{-17}^{+21} \mathrm{~km} \mathrm{~s}^{-1}$ and $59_{-9.4}^{+11} \mathrm{~km} \mathrm{~s}^{-1}$ (99.7\% CL) for the luminosities of G1 and G2, respectively. Interestingly, they agree, within the statistical errors, with the values optimized separately for $\sigma_{v}^{\mathrm{G} 1}$ and $\sigma_{v}^{\mathrm{G} 2}$ (see Table 2), thus indicating that the total intrinsic scatter in the total mass-to-light ratios of the subhalo population does not seem to play an important role in the cluster strong lensing modeling.

In Fig. 6, we compare the cumulative projected total mass profile resulting from our best-fitting strong lensing model with that obtained from the X-ray emission and dynamical analysis of $\sim 800$ cluster galaxies. Interestingly, the latter is also best described by a softened (i.e., cored) isothermal sphere model, as discussed in Balestra et al. (2016). The new strong lensing total mass profile is perfectly consistent with that of Gr15, who used $\sim 1 / 3$ of the multiple images and the GLEE (Suyu \& Halkola 2010; Suyu et al. 2012) software for the modeling. Although the sub-halo total mass distribution in our model is lower than in $\mathrm{Gr} 15$, both measurements agree within the statistical errors (99.7\% CL). This difference in mainly related to the inclusion of the third dark matter halo and the extra constraints in our strong lensing model. In the right panel, we also compare the projected total mass and the X-ray surface brightness distribution. As discussed in Balestra et al. (2016) the close resemblance of the total mass and gas component, adds further evidence to a pre-merger scenario for MACS 0416.

In addition, we compare our magnification map for a source at redshift 4 with the HFF models in Fig. 7. The models cover different methodologies: 1) free-form, meaning that no parametric form is assumed for the total mass distribution, Bradac v3 (Hoag et al. 2016), Willians v3 (using the GRALE software Liesenborgs et al. 2006, 2010) and Diego v3 (a pre-HFF modeling is presetend in Diego et al. 2015b); 2) hybrid, that scales the smooth dark matter component with the light, Zitrin-LTMGauss v3 (Broadhurst et al. 2005; Zitrin et al. 2009); 3) parametric models using different codes, Sharon v3 (see Johnson et al. 2014, for their pre-HFF modeling) and CATS v3 (pre-HFF presented in Richard et al. 2014) with lenstool and GLAFIC v3 (Kawamata et al. 2016). Differences in parametric and free-form models are expected on small spatial scales. In the case of the parametric models, the main difference is related to the northeast region due to the presence of the third halo, however the overall shape of the critical lines is very similar.

We emphasize that a full comparison of these maps becomes meaningful only when the input constraints (number and quality) of all models are the same or at least very similar. Notice that some modelers consider several knots of the same background source as different multiple image systems, increasing the number of $N_{\text {spec }}$. Moreover, as underscored in this and previous works (Gr15, Ca16, Treu et al. 2016; Rodney et al. 2016; Johnson \& Sharon 2016) a large number of spectroscopic families is critical for the robustness of the lens model, removing misidentification of multiple images and alleviating model degeneracies. Also note that the use of different selection of 

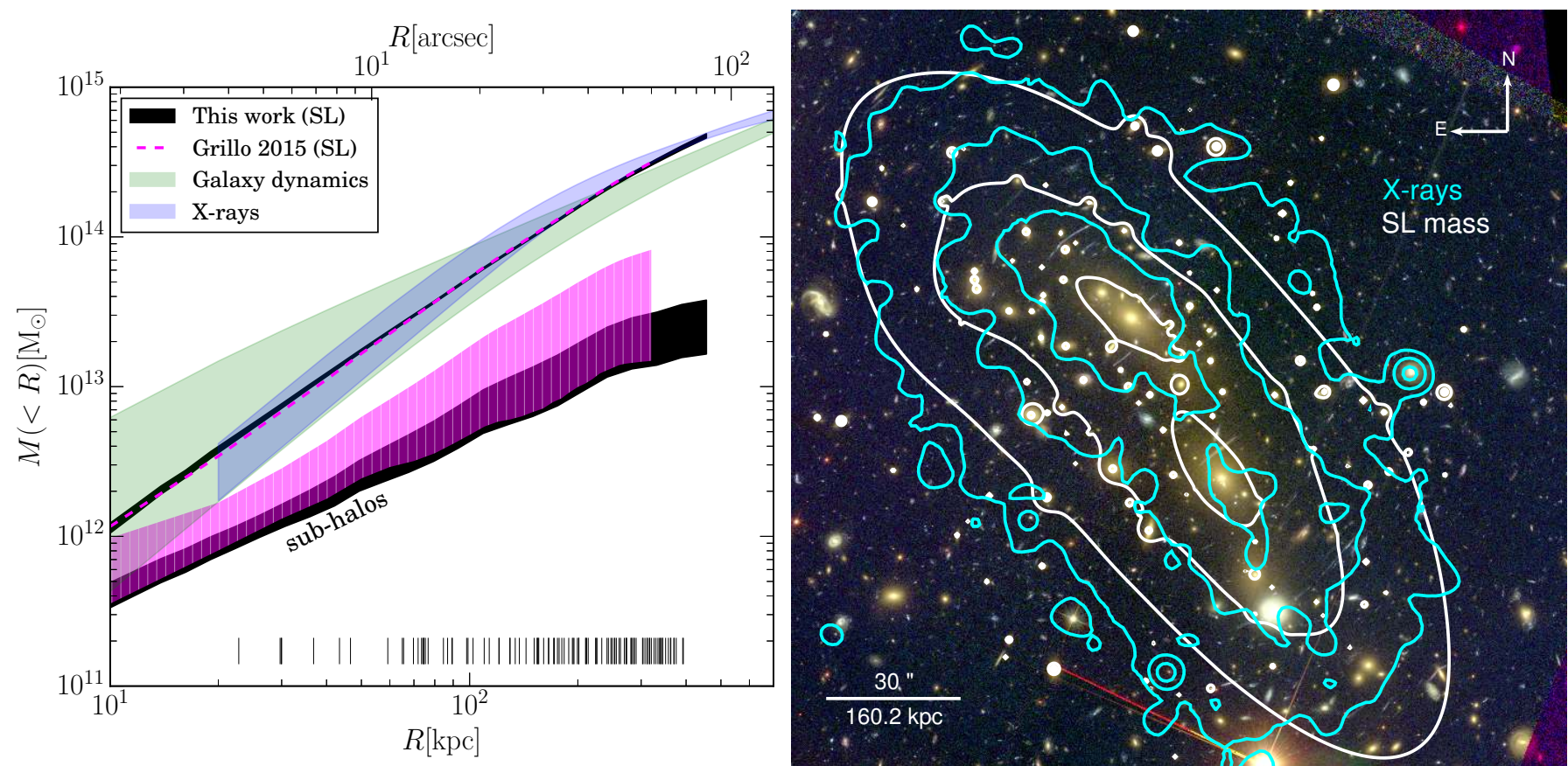

Fig. 6. Left panel: we cumulative projected mass distribution of MACS 0416 from our new strong lensing model (total mass and sub-halo component), relative to the position of the BCG, $\mathrm{N}$. The black regions correspond to the $99.7 \%$ confidence level, while the magenta line and shaded area show the best-fitting profiles from our previous model presented in Gr15. The green and blue regions are the $1 \sigma$ determination of the mass profile from the dynamical analysis of $\sim 800$ cluster galaxies and the X-ray emission, respectively, reported in Balestra et al. (2016). The total mass profiles associated to the sub-halo population of cluster galaxies from the lensing model is also shown. The vertical lines show the projected radial distances of the multiple images used in this work. Right panel: projected total mass iso-contours $\left([0.6,1,2] \times 10^{15} M_{\odot} \mathrm{Mpc}^{-2}\right.$ in white), and Chandra X-ray contours (0.5-2 KeV) overlaid on the HFF-ACS color image.

member galaxies leads to significant deviations of the magnification on small scales. In our case, a highly pure and complete sample of members is provided by MUSE spectroscopy. For a complete comparison of the HFF lensing models see Priewe et al. (2017) and Meneghetti et al. (2016) in the context of strong lensing simulations of galaxy clusters.

\section{Conclusions}

In this article, we have significantly extended the panoramic VIMOS spectroscopic campaign of MACS 0416, presented in Gr15 and Balestra et al. (2016), with data from the MUSE integralfield spectrograph on the VLT, which has yielded 208 new secure redshift measurements in the central $2 \operatorname{arcmin}^{2}$ region of the cluster. Notably, a new large set of multiply lensed sources was identified using two MUSE archival pointings, extending the work of Gr15 and Hoag et al. (2016) and bringing the number of spectroscopically identified multiple-image systems from 15 to 37 . This was possible by measuring 59 new redshifts to very faint magnitude, thanks to the sensitivity of MUSE to line fluxes as faint as $10^{-19} \mathrm{erg} \mathrm{s}^{-1} \mathrm{~cm}^{-2} \AA^{-1}$ (see Karman et al. 2017, for the study of a similar set of low-luminosity Lyman- $\alpha$ emitters with MUSE observations of the HFF cluster AS1063). This new sample also extends the redshift range of known multiple images, with five additional systems at $z>5$, one of which is at $z=6.145$ (13 images with measured redshift at $z>5$ ). The MUSE observations also allowed us to secure redshifts of 144 member galaxies over an area of $\sim 0.2 \mathrm{Mpc}^{2}$. Three-quarters of the cluster galaxies selected down to $\operatorname{mag}_{F 160 \mathrm{~W}}=24$ (corresponding to $M_{*} \approx 3 \times 10^{8} M_{\odot}$ ) are now spectroscopically confirmed.
With such a large set of 102 spectroscopic multiple images and a much improved sample of galaxy members in the cluster core, we have built a new strong lensing model and obtained an accurate determination of the projected total mass distribution of MACS 0416. The main results of this study can be summarized as follows:

1. We can reproduce the observed multiple-image positions with an accuracy of $\Delta_{\mathrm{rms}}=0 \prime .59$, which is somewhat larger than the one obtained by $\operatorname{Gr} 15\left(0^{\prime \prime} \cdot 36\right)$, who however used less than one-third of the multiple images.

2. The large-scale component of the total mass distribution was initially modeled with two cored elliptical pseudo-isothermal profiles around the two BCGs, as in Gr15, however larger positional offsets $\Delta$ in the NE portion of the cluster led us to introduce a third floating cored halo in the model. We find interesting that, besides significantly reducing the $\Delta_{\mathrm{rms}}$, the best-fit position of this third halo is very close to a peak of the convergence map obtained by Hoag et al. (2016) with an independent free-form lensing model, which also exploits the weak-lensing shear. Although this third halo is centered on a relatively small overdensity of cluster galaxies, it could not be identified in the phase-space analysis of Balestra et al. (2016), most probably because of the combination of projection effects and the absence of clear separation in the projected velocity space.

3. The new best-fitting centers of the two main halos are now found within $\sim 2^{\prime \prime}$ from the respective BCGs, further reducing the halo-BCG offset when compared with the Gr15 model. As described in Balestra et al. (2016), such a concentric distribution of light and dark-matter mass, when compared with the distribution of the X-ray emitting gas whose 


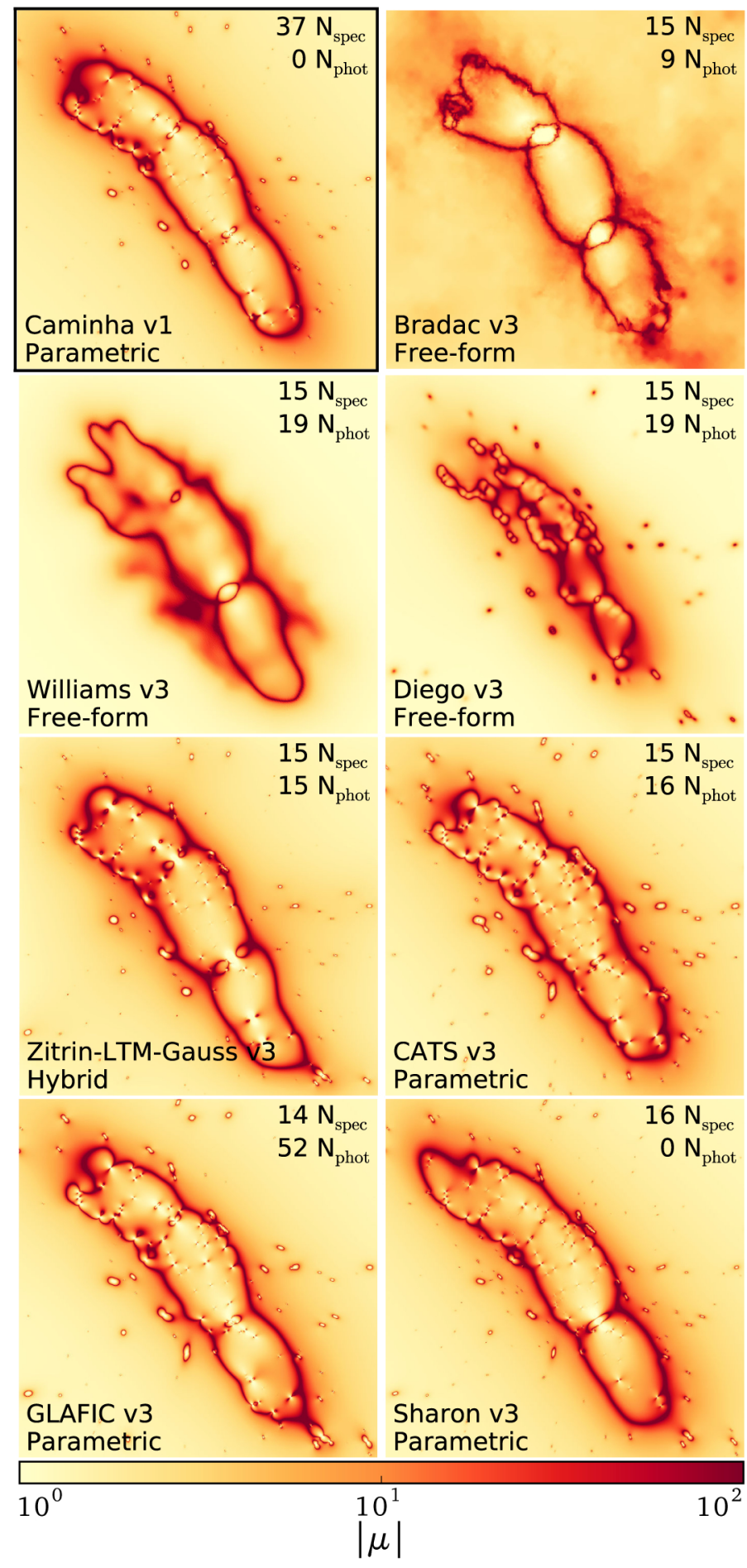

Fig. 7. Magnification maps of MACS 0416 for a source at redshift 4 We show the HFF strong lensing models to compare with the work presented here (first panel). For each model, we indicate the number of multiple image families based on spectroscopic $\left(N_{\text {spec }}\right)$ and photometric $\left(N_{\text {phot }}\right)$ information. All panels are centered at the same position and are $2 ! 1$ across.

main peak is at the position of the northern BCG, is consistent with a pre-merging scenario.

4. The cumulative projected total mass profile is found in excellent agreement with the one of $\mathrm{Gr} 15$, and in good agreement with the dynamical and X-ray mass which was however obtained with the simple approximation of a single spherical halo (see Balestra et al. 2016). Together with the point 2. above, this suggests that owing to a significant enhancement of constraints in the strong lensing model we are now able to better resolve the mass distribution of the smooth cluster halo.

5. The overall scaling of the total mass-to-light ratio for the sub-halo population, traced by the new highly complete and pure sample of cluster galaxies, is found consistent with the one of Gr15. Our new model therefore corroborates the evidence found in Gr15 that a sub-halo mass function is significantly suppressed when compared to simulations, particularly at the high-mass end. A similar result has recently been obtained in an independent study (Munari et al. 2016) of the Abell 2142 galaxy cluster with SDSS data. A detailed analysis of the sub-halo population and different mass components in the core of MACS 0416, which takes advantage of the internal velocity dispersions of cluster galaxies (see e.g., Monna et al. 2015, 2017) is deferred to a future paper, where we also plan a detailed comparison with the study of Hoag et al. (2016).

Remarkably, the new spectroscopic identifications with MUSE observations of MACS 0416 match in some cases the continuum magnitude limit of the HFF data for Lyman- $\alpha$ emitters (see also Karman et al. 2017), and complement the HST NIR GRISM spectroscopy of the GLASS survey. Not surprisingly, this cluster now becomes one of the best test bench for strong lensing modeling (see Fig. 3), which we argue need to rely largely, or entirely, on spectroscopically confirmed multiple-image systems for high-precision modeling.

The accuracy we have reached in reproducing the observed multiple-image positions with this new model, on the other hand suggests that it will be challenging to further improve on these results by simply introducing more mass components in parametric models. Interestingly, the large number of constraints for this cluster should allow free-form models to become more effective, for example in discovering extra mass clumps with unusual total mass-to-light ratios. As already noted in Ca16 (see also Treu et al. 2016), with the current high-quality set of strong lensing constraints we seem to have hit the limit of the singleplane lensing approximation, so that the next step in precision strong-lensing modeling inevitably will have to properly take into account the effects of the structure along the line of sight, adequately sampled by spectroscopic data.

As previously done with CLASH-VLT VIMOS observations of HFF clusters, we make public the new extended redshift cat$\operatorname{alog}^{2}$, which includes secure redshift determinations from the MUSE data, in the effort to add further value to the entire HFF dataset.

Acknowledgements. The authors thank the anonymous referee for the useful comments on the manuscript. We acknowledge financial support from PRININAF 2014 1.05.01.94.02. C.G. acknowledges support by VILLUM FONDEN Young Investigator Program grant 10123. This work made use of the CHE cluster, managed and funded by ICRA/CBPF/MCTI, with financial support from FINEP (grant 01.07.0515.00 from CT-INFRA - 01/2006) and FAPERJ (grants E-26/171.206/2006 and E-26/110.516/2012). P.R. acknowledges the hospitality and support of the visitor program of the DFG cluster of excellence "Origin and Structure of the Universe". This work made use of data taken under the ESO programs ID 094.A-0115(B) and ID 094.A-0525(A), and also 186.A-0798.

\footnotetext{
2 The full redshift catalog including VIMOS and MUSE measurments can be found at the CDS and at the link: https://sites.google. com/site/vltclashpublic/data-release
} 


\section{References}

Bacon, R., Accardo, M., Adjali, L., et al. 2012, The Messenger, 147, 4 Balestra, I., Mercurio, A., Sartoris, B., et al. 2016, ApJS, 224, 33 Bender, R., Burstein, D., \& Faber, S. M. 1992, ApJ, 399, 462 Bertin, E., \& Arnouts, S. 1996, A\&AS, 117, 393

Biviano, A., Rosati, P., Balestra, I., et al. 2013, A\&A, 558, A1 Bouwens, R. J., Bradley, L., Zitrin, A., et al. 2014, ApJ, 795, 126

Broadhurst, T., Benítez, N., Coe, D., et al. 2005, ApJ, 621, 53

Caminha, G. B., Grillo, C., Rosati, P., et al. 2016, A\&A, 587, A80

Castellano, M., Amorín, R., Merlin, E., et al. 2016, A\&A, 590, A31

Coe, D., Zitrin, A., Carrasco, M., et al. 2013, ApJ, 762, 32

Diego, J. M., Broadhurst, T., Benitez, N., et al. 2015a, MNRAS, 446 683

Diego, J. M., Broadhurst, T., Molnar, S. M., Lam, D., \& Lim, J. 2015b, MNRAS, 447,3130

Diego, J. M., Broadhurst, T., Wong, J., et al. 2016, MNRAS, 459, 3447

Elíasdóttir, Á., Limousin, M., Richard, J., et al. 2007, ArXiv e-prints [arXiv:0710.5636]

Faber, S. M., Dressler, A., Davies, R. L., Burstein, D., \& Lynden-Bell, D. 1987, in Nearly Normal Galaxies, From the Planck Time to the Present, 175

Falco, E. E., Gorenstein, M. V., \& Shapiro, I. I. 1985, ApJ, 289, L1

Garilli, B., Fumana, M., Franzetti, P., et al. 2010, PASP, 122, 827

Grillo, C., Suyu, S. H., Rosati, P., et al. 2015, ApJ, 800, 38

Grillo, C., Karman, W., Suyu, S. H., et al. 2016, ApJ, 822, 78

Hoag, A., Huang, K.-H., Treu, T., et al. 2016, ApJ, 831, 182

Host, O. 2012, MNRAS, 420, L18

Jauzac, M., Clément, B., Limousin, M., et al. 2014, MNRAS, 443, 1549

Jauzac, M., Jullo, E., Eckert, D., et al. 2015a, MNRAS, 446, 4132

Jauzac, M., Richard, J., Jullo, E., et al. 2015b, MNRAS, 452, 1437

Johnson, T. L., \& Sharon, K. 2016, ApJ, 832, 82

Johnson, T. L., Sharon, K., Bayliss, M. B., et al. 2014, ApJ, 797, 48

Jullo, E., Kneib, J.-P., Limousin, M., et al. 2007, New J. Phys., 9, 447

Jullo, E., Natarajan, P., Kneib, J.-P., et al. 2010, Science, 329, 924

Kahlhoefer, F., Schmidt-Hoberg, K., Kummer, J., \& Sarkar, S. 2015, MNRAS 452, L54

Karman, W., Caputi, K. I., Caminha, G. B., et al. 2017, A\&A, 599, A28

Kassiola, A., \& Kovner, I. 1993, ApJ, 417, 450

Kawamata, R., Oguri, M., Ishigaki, M., Shimasaku, K., \& Ouchi, M. 2016, ApJ, 819,114

Kelly, P. L., Rodney, S. A., Treu, T., et al. 2016, ApJ, 819, L8

Kimm, T., \& Cen, R. 2014, ApJ, 788, 121

Kneib, J.-P., Ellis, R. S., Smail, I., Couch, W. J., \& Sharples, R. M. 1996, ApJ, 471,643
Lam, D., Broadhurst, T., Diego, J. M., et al. 2014, ApJ, 797, 98

Liesenborgs, J., De Rijcke, S., \& Dejonghe, H. 2006, MNRAS, 367, 1209

Liesenborgs, J., de Rijcke, S., \& Dejonghe, H. 2010, GRALE: A genetic algorithm for the non-parametric inversion of strong lensing systems, Astrophysics Source Code Library

Limousin, M., Richard, J., Jullo, E., et al. 2007, ApJ, 668, 643

Limousin, M., Richard, J., Jullo, E., et al. 2016, A\&A, 588, A99

Lotz, J. M., Koekemoer, A., Coe, D., et al. 2016, ArXiv e-prints [arXiv: 1605.06567]

Mann, A. W., \& Ebeling, H. 2012, MNRAS, 420, 2120

Masters, D., \& Capak, P. 2011, PASP, 123, 638

Meneghetti, M., Natarajan, P., Coe, D., et al. 2016, ArXiv e-prints [arXiv: 1606.04548]

Monna, A., Seitz, S., Zitrin, A., et al. 2015, MNRAS, 447, 1224

Monna, A., Seitz, S., Geller, M. J., et al. 2017, MNRAS, 465, 4589

Munari, E., Grillo, C., De Lucia, G., et al. 2016, ApJ, 827, L5

Pizzuti, L., Sartoris, B., Borgani, S., et al. 2016, J. Cosmol. Astropart. Phys., 4, 023

Postman, M., Coe, D., Benítez, N., et al. 2012, ApJS, 199, 25

Richard, J., Jauzac, M., Limousin, M., et al. 2014, MNRAS, 444, 268

Priewe, J., Williams, L. L. R., Liesenborgs, J., Coe, D., \& Rodney, S. A. 2017, MNRAS, 465, 1030

Richard, J., Kneib, J.-P., Limousin, M., Edge, A., \& Jullo, E. 2010, MNRAS, 402, L44

Rodney, S. A., Strolger, L.-G., Kelly, P. L., et al. 2016, ApJ, 820, 50

Sartoris, B., Biviano, A., Rosati, P., et al. 2014, ApJ, 783, L11

Seitz, C., \& Schneider, P. 1997, A\&A, 318, 687

Soto, K. T., Lilly, S. J., Bacon, R., Richard, J., \& Conseil, S. 2016, MNRAS, 458,3210

Suyu, S. H., \& Halkola, A. 2010, A\&A, 524, A94

Suyu, S. H., Hensel, S. W., McKean, J. P., et al. 2012, ApJ, 750, 10

Treu, T., Schmidt, K. B., Brammer, G. B., et al. 2015, ApJ, 812, 114

Treu, T., Brammer, G., Diego, J. M., et al. 2016, ApJ, 817, 60

Umetsu, K., Medezinski, E., Nonino, M., et al. 2014, ApJ, 795, 163

Umetsu, K., Zitrin, A., Gruen, D., et al. 2016, ApJ, 821, 116

Vanzella, E., Balestra, I., Gronke, M., et al. 2017, MNRAS, 465, 3803

Wang, X., Hoag, A., Huang, K.-H., et al. 2015, ApJ, 811, 29

Williams, L. L. R., \& Saha, P. 2011, MNRAS, 415, 448

Wise, J. H., Demchenko, V. G., Halicek, M. T., et al. 2014, MNRAS, 442, 2560

Zitrin, A., Broadhurst, T., Umetsu, K., et al. 2009, MNRAS, 396, 1985

Zitrin, A., Meneghetti, M., Umetsu, K., et al. 2013, ApJ, 762, L30

Zitrin, A., Fabris, A., Merten, J., et al. 2015, ApJ, 801, 44 


\section{Appendix A: Multiple image properties and spectra}

In this appendix, we present in Table A.1 the information about the multiple images used in the strong lensing modeling. The full redshift catalog, combining the MUSE and VIMOS measurements (Table A.2), is available at the CDS of the paper, as well as at the link https://sites.google.com/ site/vltclashpublic/data-release. In Fig. A.1, we show the MUSE spectra around relevant spectral features and image cutouts for multiple images. 
Table A.1. Information on spectroscopically identified multiple images in MACS 0416.

\begin{tabular}{|c|c|c|c|c|c|c|}
\hline ID & RA & Dec & $z_{\text {MUSE }}$ & $z_{\text {previous }}$ & $\mathrm{ID}_{\text {ref }}$ & $\operatorname{mag}_{F 814 W}$ \\
\hline $1 \mathrm{a}$ & 64.049084 & -24.062862 & 3.2355 & - & 26.3 & $27.87 \pm 0.13$ \\
\hline $1 b$ & 64.046959 & -24.060797 & 3.2355 & - & 26.2 & $27.43 \pm 0.09$ \\
\hline $1 \mathrm{c}$ & 64.046449 & -24.060397 & 3.2355 & $2.185^{a}$ & 26.1 & $26.53 \pm 0.06$ \\
\hline $2 \mathrm{a}$ & 64.050865 & -24.066538 & 6.1452 & - & 207.2 & $28.20 \pm 1.09^{*}$ \\
\hline $2 b$ & 64.048179 & -24.062406 & 6.1452 & - & - & $29.06 \pm 1.09^{*}$ \\
\hline $2 \mathrm{c}$ & 64.043572 & -24.059004 & 6.1452 & - & 206.1 & $28.66 \pm 1.09^{*}$ \\
\hline $3 a$ & 64.049232 & -24.068174 & 3.2885 & - & 44.3 & $27.50 \pm 0.11$ \\
\hline $3 b$ & 64.045269 & -24.062763 & 3.2885 & - & 44.1 & $25.69 \pm 0.05$ \\
\hline $3 c$ & 64.041556 & -24.059997 & 3.2885 & - & 44.2 & $26.13 \pm 0.06$ \\
\hline $4 a$ & 64.048126 & -24.066957 & - & - & 27.1 & $25.73 \pm 0.03$ \\
\hline $4 b$ & 64.047468 & -24.066039 & - & $2.107^{a}$ & 27.2 & $24.13 \pm 0.03$ \\
\hline $4 \mathrm{c}$ & 64.042209 & -24.060541 & - & - & 27.3 & $25.81 \pm 0.04$ \\
\hline $5 a$ & 64.047463 & -24.068822 & 1.8950 & $1.893^{d, e, f}$ & 2.3 & - \\
\hline $5 b$ & 64.043071 & -24.063080 & 1.8950 & $1.893^{d, e, f}$ & 2.2 & $23.88 \pm 0.01^{*}$ \\
\hline $5 c$ & 64.041089 & -24.061806 & 1.8950 & $1.893^{d, e, f}$ & 2.1 & $23.67 \pm 0.01^{*}$ \\
\hline $6 a$ & 64.047808 & -24.070164 & 3.6065 & - & - & $28.53 \pm 0.23$ \\
\hline $6 b$ & 64.043657 & -24.064401 & 3.6065 & - & - & $26.73 \pm 0.20$ \\
\hline $6 c$ & 64.037676 & -24.060756 & 3.6065 & - & - & $28.00 \pm 0.17$ \\
\hline $7 a$ & 64.047098 & -24.071105 & - & $2.085^{d, e, f}$ & 7.3 & $28.05 \pm 0.14$ \\
\hline $7 b$ & 64.040664 & -24.063586 & 2.0881 & $2.085^{d, e, f}$ & 7.2 & $25.29 \pm 0.03$ \\
\hline $7 \mathrm{c}$ & 64.039795 & -24.063081 & 2.0881 & $2.085^{d, e, f}$ & 7.1 & $25.43 \pm 0.04$ \\
\hline $8 \mathrm{a}$ & 64.044624 & -24.071488 & - & $2.282^{a}$ & 29.3 & $25.81 \pm 0.06$ \\
\hline $8 b$ & 64.040485 & -24.066330 & - & $2.267^{b}$ & $29.2 \mathrm{a}$ & - \\
\hline $8 \mathrm{c}$ & 64.034256 & -24.062997 & - & - & 29.1 & $25.76 \pm 0.05$ \\
\hline $9 a$ & 64.045112 & -24.072341 & 3.2882 & - & 32.1 & $26.11 \pm 0.05^{*}$ \\
\hline $9 b$ & 64.040079 & -24.066738 & 3.2882 & - & 32.2 & $25.33 \pm 0.04^{*}$ \\
\hline$(9 \mathrm{c})$ & $(64.033157)$ & $(-24.062893)$ & - & - & - & - \\
\hline $10 \mathrm{a}$ & 64.044564 & -24.072092 & - & $2.094^{a}$ & 23.1 & $24.92 \pm 0.02$ \\
\hline $10 \mathrm{~b}$ & 64.039576 & -24.066623 & - & - & 23.2 & $25.20 \pm 0.03$ \\
\hline $10 \mathrm{c}$ & 64.034336 & -24.063734 & - & $2.091^{a}$ & 23.3 & $25.23 \pm 0.03$ \\
\hline (11a) & $(64.046676)$ & $(-24.075312)$ & - & - & - & - \\
\hline $11 b$ & 64.038515 & -24.065965 & 3.2922 & - & 55.3 & - \\
\hline $11 \mathrm{c}$ & 64.035223 & -24.064731 & 3.2922 & - & 55.1 & $27.93 \pm 0.11$ \\
\hline (12a) & $(64.041365)$ & $(-24.070852)$ & 0.9397 & - & 28.3 & - \\
\hline $12 b$ & 64.036843 & -24.067457 & 0.9397 & $0.937^{a}$ & 28.2 & - \\
\hline $12 \mathrm{c}$ & 64.036507 & -24.067028 & 0.9397 & $0.937^{a}$ & 28.1 & - \\
\hline $13 \mathrm{a}$ & 64.039245 & -24.070383 & 1.0054 & $1.005^{c}$ & 11.1 & - \\
\hline $13 b$ & 64.038301 & -24.069728 & 1.0054 & $1.005^{c}$ & 11.2 & - \\
\hline $13 \mathrm{c}$ & 64.034234 & -24.066016 & 1.0054 & - & 11.3 & $26.79 \pm 0.12$ \\
\hline $14 \mathrm{a}$ & 64.034483 & -24.066956 & 3.2215 & - & D22.1 & - \\
\hline $14 \mathrm{~b}$ & 64.034190 & -24.066488 & 3.2215 & - & D22.2 & - \\
\hline $14 \mathrm{c}$ & 64.034001 & -24.066445 & 3.2215 & - & D22.3 & - \\
\hline (14d) & (64.045886) & $(-24.076722)$ & - & - & - & - \\
\hline$(14 \mathrm{e})$ & (64.035165) & $(-24.067968)$ & - & - & - & - \\
\hline $15 \mathrm{a}$ & 64.041802 & -24.075731 & 1.9894 & $1.989^{d, e, f}$ & 3.3 & $23.99 \pm 0.01$ \\
\hline $15 \mathrm{~b}$ & 64.035249 & -24.070989 & 1.9894 & $1.989^{d, e, f}$ & 3.2 & $23.94 \pm 0.02$ \\
\hline $15 c$ & 64.030769 & -24.067129 & 1.9894 & $1.989^{d, e, f}$ & 3.1 & $24.17 \pm 0.01$ \\
\hline $16 a$ & 64.033523 & -24.069448 & 2.0948 & - & 5.3 & $24.11 \pm 0.02$ \\
\hline $16 b$ & 64.032656 & -24.068663 & 2.0948 & $2.092^{b}$ & 5.2 & $24.75 \pm 0.02$ \\
\hline $16 \mathrm{c}$ & 64.032410 & -24.068414 & 2.0948 & - & 5.1 & - \\
\hline$(16 \mathrm{e})$ & $(64.043470)$ & $(-24.076860)$ & - & - & - & - \\
\hline $17 \mathrm{a}$ & 64.040489 & -24.078380 & 3.9663 & - & - & $27.97 \pm 0.27$ \\
\hline $17 \mathrm{~b}$ & 64.035107 & -24.073864 & - & - & - & $25.29 \pm 0.17^{*}$ \\
\hline $17 \mathrm{c}$ & 64.027171 & -24.068224 & - & - & - & $27.47 \pm 0.17^{*}$ \\
\hline
\end{tabular}

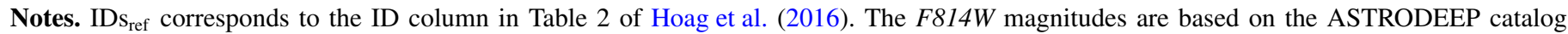
(Castellano et al. 2016). Model-predicted positions are indicated in brackets. MUSE redshifts are generally based on the Ly- $\alpha$ line when present, with exception of IDs 1, 3, 9, 20, 26, and 27 for which CIV and CIII] lines were used to better estimate the systemic redshift. This table includes 56 new redshifts belonging to 22 multiply lensed sources. ${ }^{(\dagger)}$ Positions measured in MUSE narrow band images; ${ }^{(a)}$ GLASS redshifts Hoag et al. (2016); ${ }^{(b)}$ Balestra et al. (2016); ${ }^{(c)}$ Rodney et al., in prep. ${ }^{(d)}$ Grillo et al. (2015); ${ }^{(e)}$ Jauzac et al. (2014); ${ }^{(f)}$ Richard et al. (2014); ${ }^{(g)}$ Zitrin et al. (2013); ${ }^{(*)}$ Objects whose magnitude might be affected by source confusion. 
Table A.1. continued.

\begin{tabular}{|c|c|c|c|c|c|c|}
\hline ID & RA & Dec & $z_{\text {MUSE }}$ & $z_{\text {previous }}$ & $\mathrm{ID}_{\text {ref }}$ & $\operatorname{mag}_{F 814 W}$ \\
\hline $18 \mathrm{a}$ & 64.040177 & -24.079872 & 3.8710 & - & 49.2 & $27.12 \pm 0.09$ \\
\hline $18 \mathrm{~b}$ & 64.033937 & -24.074565 & 3.8710 & - & 49.1 & $27.08 \pm 0.15$ \\
\hline$(18 \mathrm{c})$ & (64.026991) & $(-24.069620)$ & - & - & - & - \\
\hline $19 a$ & 64.040140 & -24.080305 & 4.1032 & - & 51.1 & $26.70 \pm 0.08^{*}$ \\
\hline $19 b$ & 64.033667 & -24.074762 & 4.1032 & - & 51.2 & $25.38 \pm 0.11^{*}$ \\
\hline $19 \mathrm{c}$ & 64.026633 & -24.070476 & 4.1032 & - & 51.3 & - \\
\hline $20 \mathrm{a}$ & 64.040351 & -24.081482 & - & $3.223^{d, e, f}$ & 13.3 & $25.75 \pm 0.03$ \\
\hline $20 \mathrm{~b}$ & 64.032157 & -24.075108 & 3.2175 & $3.223^{d, e, f}$ & 13.2 & - \\
\hline $20 c$ & 64.027572 & -24.072673 & 3.2175 & $3.223^{d, e, f}$ & 13.1 & $24.64 \pm 0.02$ \\
\hline (21a) & $(64.042028)$ & $(-24.081835)$ & - & - & - & - \\
\hline $21 b^{\dagger}$ & 64.030906 & -24.074341 & 5.1060 & - & 34.2 & - \\
\hline $21 c^{\dagger}$ & 64.029176 & -24.073382 & 5.1060 & - & 34.1 & - \\
\hline (22a) & $(64.040114)$ & $(-24.082217)$ & - & - & - & - \\
\hline $22 b$ & 64.030997 & -24.077173 & 3.9230 & - & 60.3 & - \\
\hline $22 c$ & 64.027127 & -24.073572 & 3.9230 & - & - & $29.54 \pm 0.43$ \\
\hline $23 a$ & 64.035668 & -24.079920 & 2.5425 & $2.545^{b}$ & 45.1 & $26.27 \pm 0.05$ \\
\hline $23 b$ & 64.032638 & -24.078508 & 2.5425 & - & - & - \\
\hline$(23 c)$ & (64.024668) & $(-24.071076)$ & - & - & - & - \\
\hline $24 a$ & 64.035833 & -24.081321 & 1.6333 & $1.637^{d}$ & 14.3 & $23.19 \pm 0.01$ \\
\hline $24 \mathrm{~b}$ & 64.031039 & -24.078953 & 1.6333 & $1.637^{d}$ & 14.2 & $23.34 \pm 0.01$ \\
\hline $24 \mathrm{c}$ & 64.026239 & -24.074337 & 1.6333 & $1.637^{d}$ & 14.1 & $23.41 \pm 0.01$ \\
\hline $25 a$ & 64.038073 & -24.082404 & 3.1103 & - & 67.1 & $27.84 \pm 0.13^{*}$ \\
\hline $25 b$ & 64.030366 & -24.079015 & 3.1103 & - & 67.3 & - \\
\hline $25 \mathrm{c}$ & 64.025446 & -24.073648 & 3.1103 & - & 67.2 & $28.06 \pm 0.13$ \\
\hline $26 a$ & 64.037722 & -24.082388 & 3.0773 & - & 58.2 & $27.58 \pm 0.09$ \\
\hline $26 b$ & 64.030484 & -24.079222 & 3.0773 & - & 58.3 & $26.49 \pm 0.08$ \\
\hline $26 c$ & 64.025186 & -24.073575 & 3.0773 & - & 58.1 & $27.46 \pm 0.08$ \\
\hline $27 a$ & 64.037469 & -24.083657 & 3.4909 & - & 35.1 & - \\
\hline $27 b$ & 64.029409 & -24.079889 & 3.4909 & - & 35.2 & $26.37 \pm 0.05$ \\
\hline $27 \mathrm{c}$ & 64.024946 & -24.075021 & 3.4909 & - & 35.3 & $26.43 \pm 0.04$ \\
\hline $28 \mathrm{a}$ & 64.038350 & -24.084126 & - & - & - & $28.76 \pm 0.26$ \\
\hline $28 \mathrm{~b}$ & 64.028322 & -24.079004 & 3.2526 & - & 47.2 & - \\
\hline $28 \mathrm{c}$ & 64.026330 & -24.076705 & 3.2526 & - & 47.1 & $25.93 \pm 0.05$ \\
\hline $29 a$ & 64.036702 & -24.083855 & - & $2.298^{d, e, f}$ & 10.3 & $25.60 \pm 0.03$ \\
\hline $29 b$ & 64.028504 & -24.079755 & - & $2.298^{d, e, f}$ & 10.2 & $24.99 \pm 0.02$ \\
\hline $29 \mathrm{c}$ & 64.025993 & -24.077080 & - & $2.298^{d, e, f}$ & 10.1 & $24.57 \pm 0.02$ \\
\hline $30 \mathrm{a}$ & 64.033628 & -24.083185 & 3.4406 & - & 38.1 & $27.20 \pm 0.08$ \\
\hline $30 \mathrm{~b}$ & 64.031251 & -24.081904 & 3.4406 & - & 38.2 & $26.98 \pm 0.10^{*}$ \\
\hline $30 \mathrm{c}$ & 64.022699 & -24.074595 & 3.4406 & - & 38.3 & $27.94 \pm 0.14^{*}$ \\
\hline $31 \mathrm{a}$ & 64.035486 & -24.084679 & 4.1218 & - & 48.1 & $26.21 \pm 0.08$ \\
\hline $31 \mathrm{~b}$ & 64.029234 & -24.081813 & 4.1218 & - & 48.2 & $24.76 \pm 0.06^{*}$ \\
\hline $31 \mathrm{c}$ & 64.023412 & -24.076125 & 4.1218 & - & 48.3 & $25.39 \pm 0.06$ \\
\hline $32 a$ & 64.035054 & -24.085504 & 5.3650 & - & 33.2 & $27.95 \pm 1.09$ \\
\hline $32 b$ & 64.028403 & -24.082993 & 5.3650 & - & 33.1 & $26.60 \pm 0.70^{*}$ \\
\hline $32 \mathrm{c}$ & 64.022988 & -24.077265 & 5.3650 & - & 33.2 & $26.03 \pm 0.20^{*}$ \\
\hline $33 a$ & 64.032017 & -24.084230 & 5.9729 & - & - & $27.03 \pm 0.82^{*}$ \\
\hline $33 b$ & 64.030821 & -24.083697 & 5.9729 & - & - & - \\
\hline (33c) & (64.021697) & $(-24.075230)$ & - & - & - & - \\
\hline (34a) & $(64.034067)$ & $(-24.085284)$ & - & - & - & - \\
\hline $34 \mathrm{~b}$ & 64.027632 & -24.082609 & 3.9228 & - & - & $27.57 \pm 0.28$ \\
\hline $34 \mathrm{c}$ & 64.023731 & -24.078477 & 3.9228 & - & - & $28.19 \pm 0.45$ \\
\hline $35 a^{\dagger}$ & 64.033681 & -24.085855 & 5.6390 & - & - & - \\
\hline $35 b^{\dagger}$ & 64.028654 & -24.084240 & 5.6390 & - & - & - \\
\hline $35 c^{\dagger}$ & 64.022187 & -24.077559 & 5.6390 & - & - & - \\
\hline $36 a$ & 64.031614 & -24.085762 & - & $1.964^{d, e}$ & 16.3 & $24.35 \pm 0.02$ \\
\hline $36 \mathrm{~b}$ & 64.028339 & -24.084553 & - & $1.964^{d, e}$ & 16.2 & $23.48 \pm 0.02$ \\
\hline $36 \mathrm{c}$ & 64.024074 & -24.080895 & 1.9614 & $1.964^{d, e}$ & 16.1 & - \\
\hline $37 a$ & 64.029809 & -24.086363 & - & $2.218^{d, e, f}$ & 17.1 & $24.32 \pm 0.01$ \\
\hline $37 \mathrm{~b}$ & 64.028610 & -24.085973 & - & $2.218^{d, e, f}$ & 17.2 & - \\
\hline $37 \mathrm{c}$ & 64.023345 & -24.081580 & 2.2182 & $2.218^{d, e, f}$ & 17.3 & - \\
\hline
\end{tabular}


Table A.2. CLASH-VLT and MUSE spectroscopic redshift catalog of MACS J0416.

\begin{tabular}{|c|c|c|c|c|c|c|}
\hline $\begin{array}{l}\text { ID } \\
(1)\end{array}$ & $\begin{array}{l}\text { RA } \\
(2)\end{array}$ & $\begin{array}{c}\text { Dec } \\
(3)\end{array}$ & $\begin{array}{c}z \\
(4)\end{array}$ & $\begin{array}{l}\text { QF } \\
(5)\end{array}$ & $\begin{array}{c}\text { Ref. } \\
\text { (6) }\end{array}$ & $\begin{array}{c}\text { Mag } \\
\text { (7) }\end{array}$ \\
\hline CLASHVLTJ041512.49-240506.6 & 63.802059 & -24.085165 & 0.5772 & 3 & 1 & 22.17 \\
\hline CLASHVLTJ041512.65-240924.4 & 63.802703 & -24.156778 & 0.4043 & 3 & 1 & 22.71 \\
\hline CLASHVLTJ041512.65-240653.9 & 63.802720 & -24.114978 & 0.3032 & 3 & 1 & 18.48 \\
\hline CLASHVLTJ041512.67-240500.3 & 63.802779 & -24.083429 & 0.5767 & 3 & 1 & 21.97 \\
\hline CLASHVLTJ041512.69-241059.8 & 63.802859 & -24.183278 & 2.9782 & 9 & 1 & 23.24 \\
\hline CLASHVLTJ041512.75-240625.2 & 63.803134 & -24.107008 & 0.6372 & 3 & 1 & 23.56 \\
\hline CLASHVLTJ041512.77-240106.8 & 63.803218 & -24.018566 & 0.3102 & 3 & 1 & 20.85 \\
\hline CLASHVLTJ041512.81-241112.8 & 63.803373 & -24.186895 & 0.4004 & 3 & 1 & 21.43 \\
\hline CLASHVLTJ041512.82-241351.8 & 63.803418 & -24.231058 & 0.5361 & 3 & 1 & 20.65 \\
\hline CLASHVLTJ041512.82-241417.2 & 63.803430 & -24.238104 & 0.5788 & 3 & 1 & 23.34 \\
\hline CLASHVLTJ041512.85-241308.4 & 63.803547 & -24.219004 & 0.3007 & 9 & 1 & 24.51 \\
\hline CLASHVLTJ041512.86-235617.4 & 63.803602 & -23.938160 & 0.3436 & 3 & 1 & 21.45 \\
\hline CLASHVLTJ041513.01-241423.8 & 63.804188 & -24.239932 & 0.3398 & 3 & 1 & 22.09 \\
\hline CLASHVLTJ041513.02-240640.1 & 63.804258 & -24.111131 & 0.3046 & 3 & 1 & 20.48 \\
\hline CLASHVLTJ041513.11-241022.7 & 63.804605 & -24.172971 & 0.4204 & 2 & 1 & 22.06 \\
\hline$\vdots$ & . & & $\vdots$ & $\vdots$ & $\vdots$ & $\vdots$ \\
\hline
\end{tabular}

Notes. The first 15 entries of the redshift table. The full catalog is available at the CDS and in the link https://sites.google.com/site/ vltclashpublic/data-release, and contains 7 columns and 4594 rows. The columns correspond to: (1) a unique identification reference; (23 ) the coordinates in degree; (4) the spectroscopic redshift; (5) the redshift quality flag; (6) the redshift reference (1-CLASH-VLT VIMOS based on LR-Blue spectra, 2-CLASH-VLT VIMOS based on MR spectra, 4-from Ebeling et al. (2014), 5-from Magellan, D. Kelson (priv. comm.), 6 -from VLT/MUSE, this work) and (7) is the Subaru $R$-band magnitude. 

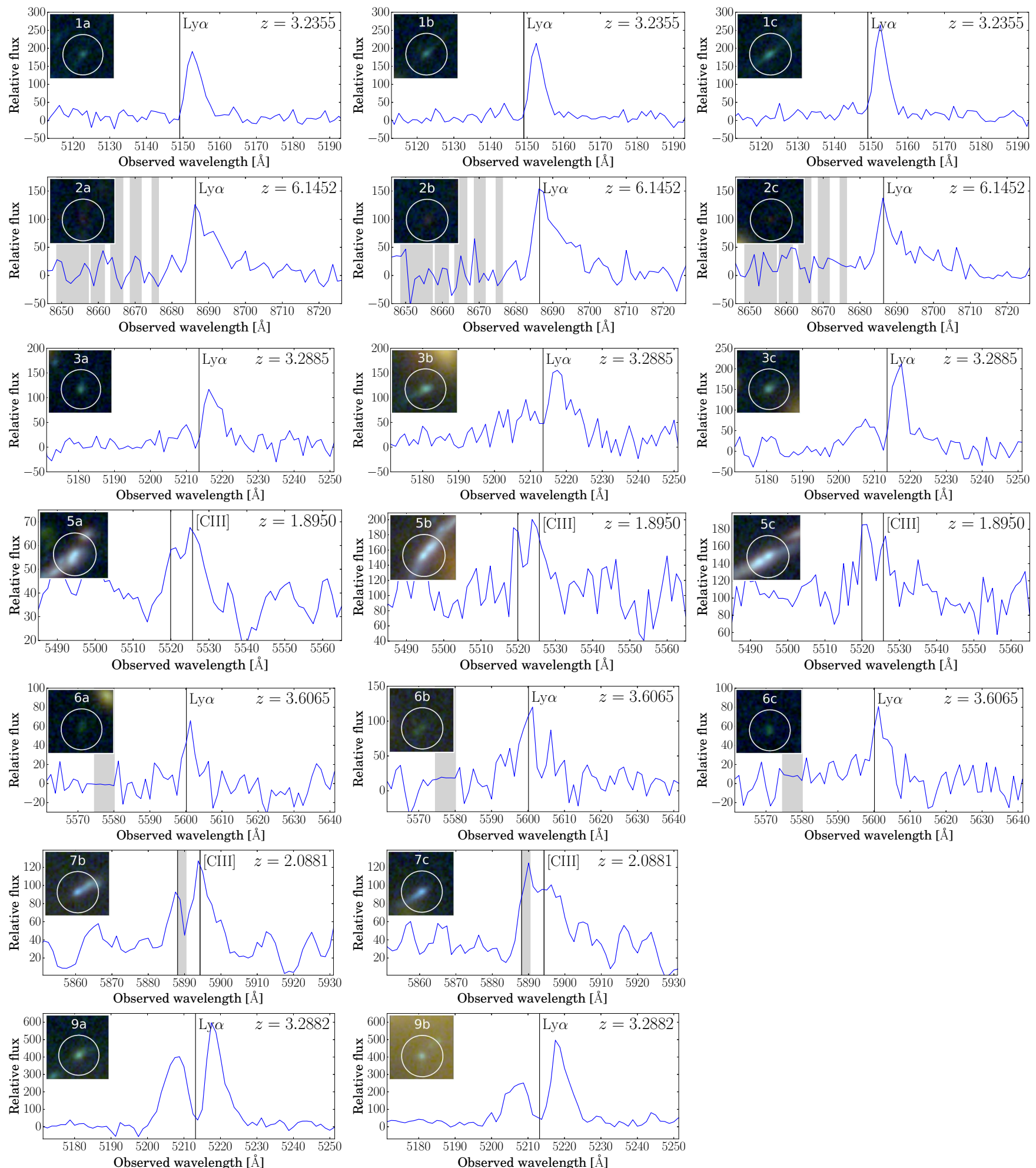

Fig. A.1. MUSE spectra of multiple images of lensed sources in MACS 0416. Panels on the same line show the spectra of multiple images belonging to the same family, i.e., associated to the same source. The vertical black lines indicate the position of the emission line based on the best estimate of the systemic redshift (see Table A.1 notes). Spectral regions with high sky contamination are marked in grey; the flux is given in units of $10^{-20} \mathrm{erg} \mathrm{s}^{-1} \mathrm{~cm}^{-2} \AA^{-1}$. The image cutouts in each panel (2" across) are extracted from the HFF color image and show the HST counterparts, or are centered at the position of the MUSE emission in the cases of no apparent counterparts (see families 21 and 35). 
G. B. Caminha et al.: A refined mass distribution of the cluster MACS J0416.1-2403
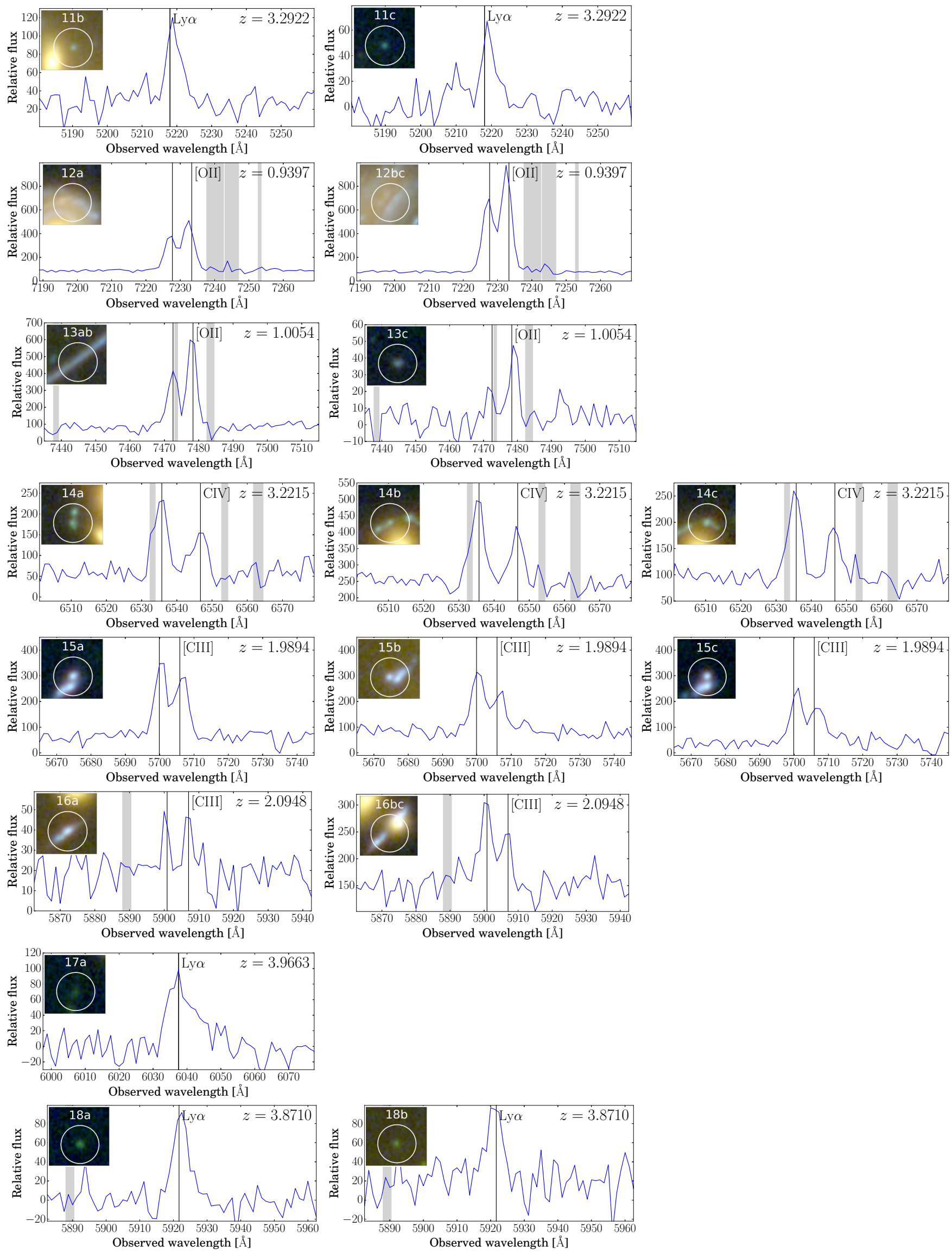

Fig. A.1. continued. 
A\&A 600, A90 (2017)
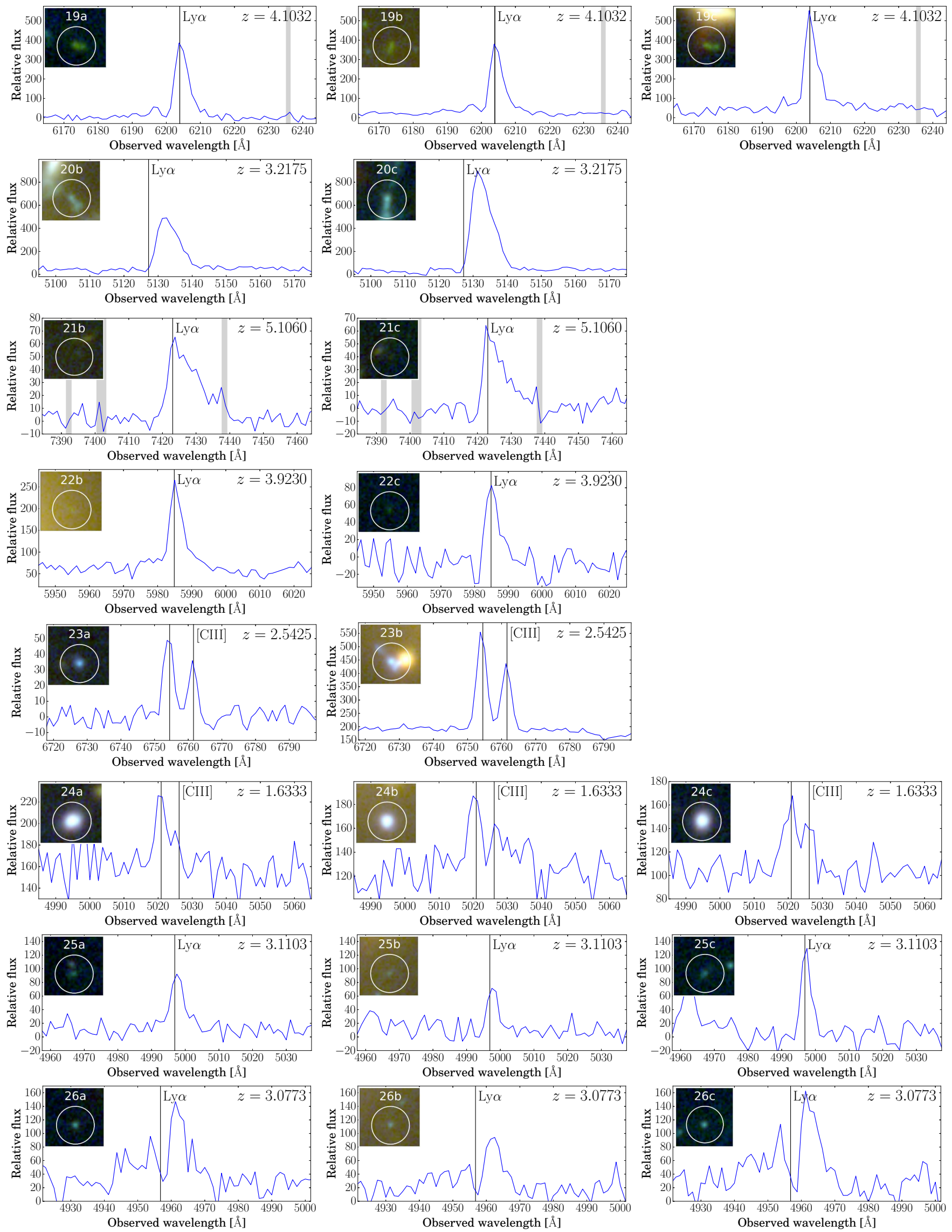

Fig. A.1. continued. 
G. B. Caminha et al.: A refined mass distribution of the cluster MACS J0416.1-2403
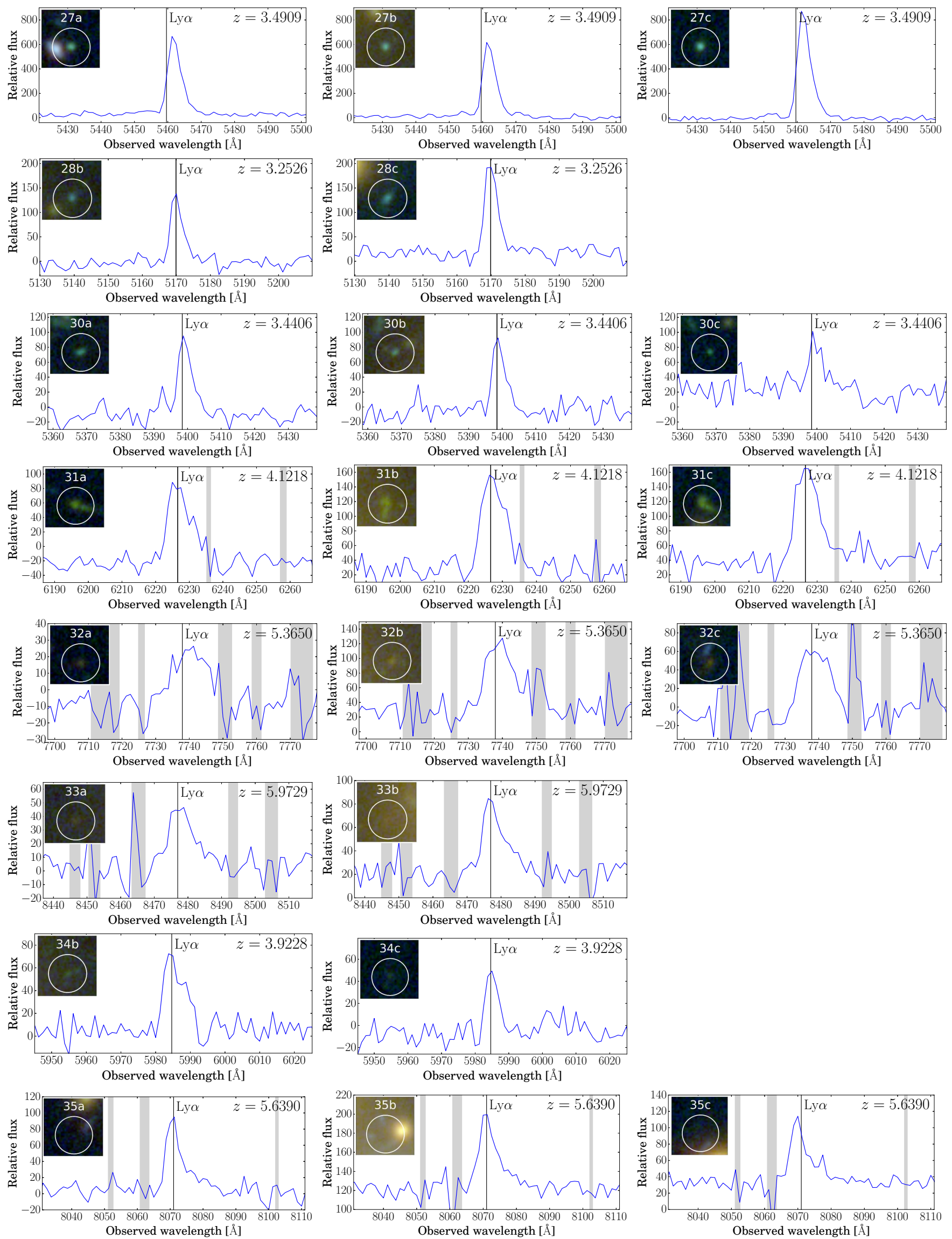

Fig. A.1. continued. 
A\&A 600, A90 (2017)
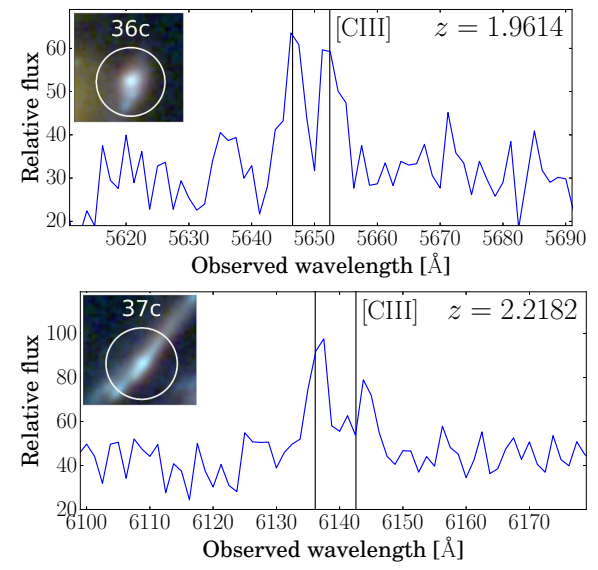

Fig. A.1. continued. 